University of New Mexico

UNM Digital Repository

Branch Mathematics and Statistics Faculty and Staff Publications

$9-8-2000$

\title{
Dynamical properties of forced shear layers in an annular geometry
}

Evangelos A. Coutsias

Keith Bergeron

J.P. Lynov

A.H. Nielsen

Follow this and additional works at: https://digitalrepository.unm.edu/math_fsp

Part of the Mathematics Commons

\section{Recommended Citation}

Journal of Fluid Mechanics, 402: 255-289

This Article is brought to you for free and open access by the Branch Academic Departments at UNM Digital Repository. It has been accepted for inclusion in Branch Mathematics and Statistics Faculty and Staff Publications by an authorized administrator of UNM Digital Repository. For more information, please contact disc@unm.edu. 


\title{
Dynamical properties of forced shear layers in an annular geometry
}

\author{
By K. BERGERON'1, E. A. COUTSIAS ${ }^{2}$, J. P. LYNOV L $^{3}$ \\ AND A. H. NIELSEN ${ }^{3}$ \\ ${ }^{1}$ Department of Mathematical Sciences, US Air Force Academy, \\ Colorado Springs, CO 80840, USA \\ ${ }^{2}$ Department of Mathematics and Statistics, University of New Mexico, \\ Albuquerque, NM 87131, USA \\ ${ }^{3}$ Association Euratom-Risø National Laboratory, Optics and Fluid Dynamics Department, \\ DK-4000 Roskilde, Denmark
}

(Received 23 February 1998 and in revised form 16 August 1999)

Results of numerical simulations of a forced shear flow in an annular geometry are presented. The particular geometry used in this work reduces the effects of centrifugal and Coriolis forces. However, there are still a large number of system parameters (shear width, shear profile, radius of curvature, initial conditions, etc.) to characterize. This set of variables is limited after the code has been validated with experimental results (Rabaud \& Couder 1983; Chomaz et al. 1988) and with the associated linear stability analysis. As part of the linear stability characterization, the pseudo-spectrum for the associated Orr-Sommerfeld operator for plane, circular Couette flow is calculated, and it is found to be insensitive to perturbations.

The numerical simulation code is a highly accurate de-aliased spectral method which utilizes banded operators to increase the computational efficiency. Viscous dissipation terms enter the code directly from the equations of motion. The results from the simulation code at low Reynolds numbers are compared with the results from linear stability analysis and are used to give predictions for the coefficients of the Landau equation describing the saturation behaviour near the critical Reynolds number. Numerical results at higher Reynolds numbers demonstrate the effects of spin-up and spin-down, including the experimentally observed hysteresis. The properties of twodimensional shears at high Reynolds numbers, at which temporal states are formed, are also addressed.

\section{Introduction}

Recent advances in spectral numerical techniques (Coutsias \& Lynov 1991; Coutsias, Hagstrom \& Torres 1995) and the pseudo-spectra of operators (Reddy, Schmid \& Henningson 1993) have reinvigorated modal analysis methods for fluid instability studies. We will show how these tools have generated new insights into circular shear flows in thin layers of a viscous, incompressible fluid. These flows, like Taylor-Couette flows, exhibit fundamental transition behaviour involving symmetry breaking, period doubling and oscillating states. Our results not only capture these states, including the hysteresis effects found in experiments, but also offer a new physical interpretation of the mode transitions during both spin-up and spin-down in terms of global mini- 
mization of energy and enstrophy. The paper concludes with an account of numerical simulations performed in regimes that have so far been experimentally inaccessible.

The particular flows evolve from the initial Kelvin-Helmholtz instability of a shear flow with a point of inflection, and they have been seen in many astrophysical, geophysical, plasma, and laboratory flows. Previous experimental, theoretical, and numerical studies reflect this variety of flows, and hence the studies have been conducted over a very wide parameter range. We have chosen to limit our analysis and simulations to a particular parameter regime in which the centrifugal and Coriolis forces do not dominate the flow. We may then take advantage of the imposed quantization and symmetry of this geometry to concentrate our efforts on the evolution of a two-dimensional shearing flow. A short review of previous studies not only illustrates the fundamental nature and importance of our results, but it also provides an account of the complications that have arisen in accurately simulating these flows.

Experimental work has included systems with rotating fluids (Rabaud \& Couder 1983; Niino \& Misawa 1984; Antonova et al. 1987; Chomaz et al. 1988; Nezlin \& Snezhkin 1993; Solomon, Holloway \& Swinney 1993), quasi-neutral plasmas (Huld et al. 1991), magnetohydrodynamic forcings (Dovzhenko \& Krymov 1987; Krymov 1988), and non-neutral plasmas (Driscoll \& Fine 1990; Peurrung \& Fajans 1993). While all of theses studies have produced similar vortical structures, the physical process and particular geometry of each apparatus differ substantially.

Whereas the experiments of Antonova et al. (1987) and Nezlin \& Snezhkin (1993) worked with an open top (i.e. a free surface), Rabaud \& Couder (1983), Chomaz et al. (1988) and Niino \& Misawa (1984) used cylindrical tanks with lids. The distinguishing feature between the French and Japanese experiments is the mechanism for inducing the shear. Rabaud \& Couder (1983) and Chomaz et al. (1988) addressed flows created by the overlap of the Ekman layers associated with the top and bottom walls of a cylindrical tank. The corresponding aspect ratio for this setup was $6.5 \leqslant \Gamma=r_{s} / h \leqslant$ 12 , where $r_{s}$ represents the shear radius and $h$ the height of the tank. They were able to catalogue an array of fundamental transition behaviours, but their linear stability analysis and numerical simulations only captured the qualitative features of the flows. Niino \& Misawa (1984) investigated barotropic instabilities in a tank with a relatively small aspect ratio, $\Gamma \approx 2$. Their apparatus was patterned after the device used by Hide \& Titman (1967) and created a two-dimensional shear as predicted by the Taylor-Proudman theorem. Their weakly nonlinear stability analysis had a strong correlation with the experimental data, but they did not report any oscillating states. From a numerical simulations point of view, it is also worth noting the difference between the annular geometry of both Rabaud \& Couder (1983) and Chomaz et al. (1988) and the disk geometry of Niino \& Misawa which introduces a coordinate singularity at the origin. In another set of experiments in an annular geometry, Solomon et al. (1993) have shown similar vortical structures. However, their work focused on behaviour that is strongly influenced by the Coriolis force and therefore mixes the Kelvin-Helmholtz instability with Rossby waves.

The disk geometry also arises naturally in the plasma and magnetohydrodynamic experiments. The work of Huld et al. (1991), which provided the original motivation for our studies, detected coherent, vortical structures in a magnetized, low-temperature plasma experiment, known as a 'Q-machine', at Risø. These vortices were created as nonlinear saturated states of the cross-field electrostatic Kelvin-Helmholtz instability in a strongly magnetized, quasi-neutral plasma. However, the statistical nature of the experimental data sampling prohibits direct and quantitative comparisons between the experiment and numerical simulations (Pécseli et al. 1992). The magnetohydro- 
dynamically forced flows employ an external, as opposed to mechanical, mechanism for creating the shear. This feature also gives the experimenter an easy method to introduce a shock excitation. However, these experimental designs also require an open top for the cylindrical tank, and this configuration introduces anti-symmetric boundary layers (Pedlosky 1987) affecting the two-dimensionality of the interior flow. The non-neutral plasma experiments of Driscoll \& Fine (1990) and Peurrung \& Fajans (1993) investigated inviscid fluids in a small aspect ratio $(\Gamma \approx 3.5)$ geometry. While the initial vortex formation follows the Kelvin-Helmholtz instability, they noted that due to the very long times required for the vortices to interact, small initial variations in the base state dictate a statistical description of subsequent vortex interactions.

In addition to numerical studies that have accompanied some of the experimental work (Niino \& Misawa 1984; Chomaz et al. 1988), there have been several independent efforts which have used computational methods to further the understanding of the shear profile and vortex pairing (Michalke 1964; Maslowe 1977; Niino 1982; Marcus 1990; Tomansini, Dolez \& Leorat 1996). Chomaz et al. (1988), Marcus (1990), and Tomansini et al. (1996) used pseudo-spectral techniques to simulate the essential physics of the flows. However, in each case the researchers presented relatively low-resolution results as a result of various implementation constraints. Chomaz et al. (1988) lost much of their resolution because of the masking method they employed to match the boundary conditions in an annular geometry. They also introduced their dissipation through an artificial hyperviscosity term. In contrast to the French simulations, Marcus (1990) imposed dissipation effects by direct removal of energy from the highest wavenumber modes. To help overcome the high-viscosity effects due to the low resolution of their simulations, Tomansini et al. (1996) maintained the shear by introducing the artificial forcing

$$
F_{s}=-v\left[\frac{\partial^{2} V}{\partial r^{2}}+\frac{\partial V}{r \partial r}-\frac{V}{r^{2}}\right]
$$

where $V$ represents the tangential velocity. In all three simulations, the qualitative nature of the initial instability and some of the aspects of the subsequent evolution of the flow with increasing Reynolds number were captured.

The lack of spectral resolution in the previous studies also plays a significant role as finer and finer vortical scales are introduced with increasing Reynolds number. In order to address this problem, we have developed very accurate and efficient Poisson and Helmholtz solvers, and for the no-slip boundary conditions a set of precomputed solvability constraints. Together with a complete de-aliasing of convolution terms and a third-order-accurate time integration scheme, these solvers enable us to achieve high-resolution results $(512 \times 256$ Fourier-Chebyshev modes $)$. We use the time evolution of the enstrophy as one of the global accuracy checks in monitoring these calculations since the enstrophy of the flow is particularly sensitive (Marcus 1990) to the introduction of these small-scale structures.

The remaining numerical studies cited have dealt with various aspects of the stability analysis of shear flows using numerical integration or finite-difference techniques. While Michalke (1964) worked with the Rayleigh stability equation of inviscid linearized stability theory with a hyperbolic-tangent velocity profile and Maslowe (1977) included the effects of Ekman friction in the inviscid equations, it was not until Niino (1982) and Niino \& Misawa (1984) combined the Ekman friction term with the viscous equations of motion that relatively good agreement was achieved between predicted and observed critical Reynolds numbers. We will describe this agreement in more detail using pseudo-spectral methods, and give the first account of the associ- 
ated linear operators. Using these linear stability results to validate our code, we then use the simulation code to estimate the Landau coefficients describing the saturation of the linearly unstable mode.

A purely theoretical study of symmetry-breaking bifurcations in a circular shear layer was presented by Churilov \& Shukhman (1992). The aim of their study was the modelling of transitions in which the mode number of the flow perturbation was reduced by one. Their analysis was based on fairly restrictive assumptions including inviscid flow, large curvature of the shear layer, and a low mode number of the perturbations. These assumptions are not fulfilled by the flows studied in the present paper.

In $\S 2$ we present the physical context of the problem we have chosen to study and the equations governing the flow. Section 3 includes a detailed description of our asymptotic analysis. We obtain the critical Reynolds number at which the axisymmetric flow, present at low Reynolds numbers, undergoes a transition into a state with a well-defined non-zero azimuthal mode number, and we investigate the sensitivity of the eigenvalues involved in this analysis to numerical truncation errors. We conclude this section with a derivation of the second- and third-order variational problems. Solvability of the third-order problem leads to a Landau equation for the slow-time-scale saturation behaviour of the linearly unstable eigenmode. Tracking the nonlinear saturation of this eigenmode in direct simulations allows us to estimate the coefficients in the various terms of the Landau equation numerically. This allows us to verify that the asymptotic theory is in very good agreement with the direct simulations for a surprisingly wide range of Reynolds numbers above the critical value. The numerical simulation code, including accuracy checks, is briefly discussed in $\S 4$. In $\S 5$, we begin by validating our computational work with results showing the agreement of the code with the linear stability analysis and experimental data. The remainder of the section focuses on the flow behaviour for specific fixed Reynolds numbers as well as spin-up and spin-down. Finally, in $\S 6$ we give some concluding remarks.

\section{Basic equations}

The physical context for our analysis is provided by the work of Rabaud \& Couder (1983) and Chomaz et al. (1988). They studied flows at the mid-plane of a cylindrical split-annulus tank with a geometry similar to one shown in figure 1 . The tank has a very small height, $h$, and the top and bottom are split at radius $a$ into two annuli. The inner and outer annuli of the top and bottom are connected through inner and outer vertical walls, with respective radii $r^{-}$and $r^{+}$, so that they can be made to rotate independently, with the outer pair rotating at angular velocity $\Omega_{2}$ and the inner pair at $\Omega_{1}$. Physical arguments are presented by Chomaz et al. (1988) showing that the flow at the mid-plane to good approximation can be considered two-dimensional. One of the objectives of the present paper is to investigate to what extent this approximation can be justified by comparisons between experimental results and well-resolved, spectral solutions to the two-dimensional equations.

In the mid-plane the shear zone separating the two differentially rotating regions has a width comparable to $h$. The main control parameter characterizing the flow is the Reynolds number,

$$
R e=\frac{\Delta \Omega a h}{v},
$$




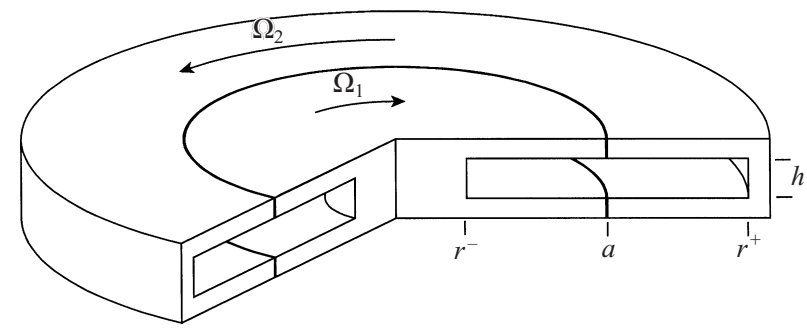

FIGURE 1. Diagram of the experimental setup used as test case.

where $\Delta \Omega=\left|\Omega_{2}-\Omega_{1}\right|$ and $v$ is the kinematic viscosity. The cell aspect ratio, $\Gamma=a / h$, also plays an important role in determining the transitions, since $\Gamma$ is a measure of how important shear curvature is, with smaller values corresponding to flows where curvature effects are more pronounced.

Following Chomaz et al. (1988), the flow in the mid-plane of the experimental setup can be approximated by the two-dimensional Navier-Stokes equations with an external Ekman forcing. Neither centifugal nor Coriolis forces have an influence on the flow as long as the cell height, $h$, is smaller than, or of the same magnitude as, the Ekman layer thickness, $e \approx\left(v /\left|\Omega_{2}-\Omega_{1}\right|\right)^{1 / 2}$, which was satisfied in the laboratory experiments. In the vorticity-stream function formulation, the governing equations are the vorticity evolution equation

$$
\frac{\partial \omega}{\partial t}+J(\omega, \psi)=v \nabla^{2} \omega+\frac{8 v}{h^{2}}\left(\omega^{*}-\omega\right),
$$

and the Poisson equation

$$
\nabla^{2} \psi=-\omega .
$$

In these equations, we have introduced the scalar vorticity $\omega$

$$
\omega \hat{\boldsymbol{e}}_{z}=\omega=\nabla \times \boldsymbol{u},
$$

the stream function $\psi$

$$
\nabla \psi \times \hat{\boldsymbol{e}}_{z}=\boldsymbol{u}
$$

and the Jacobian $J(f, g)$

$$
J(f, g) \equiv \frac{1}{r}\left(\frac{\partial f}{\partial r} \frac{\partial g}{\partial \theta}-\frac{\partial g}{\partial r} \frac{\partial f}{\partial \theta}\right) .
$$

In (2.2), the Ekman forcing term $8 v / h^{2}\left(\omega^{*}-\omega\right)$ represents the effect of the drag, $v \partial^{2} \boldsymbol{U}_{h} / \partial z^{2}$, in the central plane due to friction from the horizontal walls. In modelling this drag, it is assumed that the horizontal velocity, $\boldsymbol{U}_{h}$, has a parabolic vertical profile corresponding to a Poiseuille flow between the horizontal walls located at $z= \pm h / 2$. We note that in the paper by Chomaz et al. (1988) there is an obvious misprint in equation (3), where the forcing term in the vorticity equation is printed with $\psi$ rather than $\omega$.

The external forcing function, $\omega^{*}$, in (2.2) is an axisymmetric function that models the experimental conditions by matching the angular velocities $\Omega_{1}$ and $\Omega_{2}$ and by providing some functional relation for the transition between these two values over the shear layer. In Chomaz et al. (1988) the dynamical equations were expressed in primitive variables, so the forcing function was described in terms of the azimuthal 

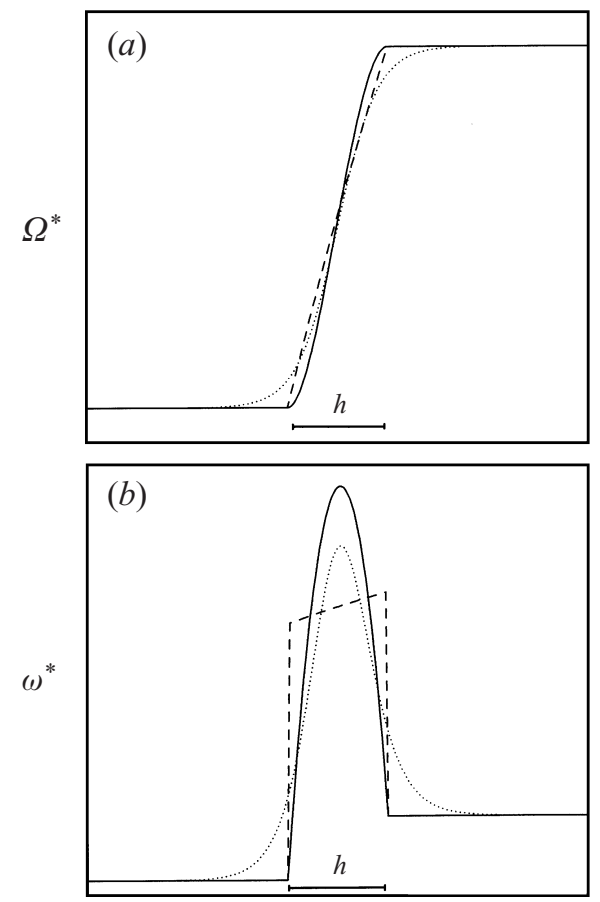

FIGURE 2. Different shapes of the forcing function in the transition region. (a) Angular velocity $\Omega^{*}(r) ;(b)$ vorticity $\omega^{*}(r)$. Dashed lines show linear interpolation in $\Omega^{*}$, while solid lines show cubic interpolation. Dotted lines show a $\tanh (r / \Delta r)$ relation for $\Omega^{*}$ in which the ratio of $\Delta r$ to the shear layer width, $h$, is $\Delta r / h=0.4$.

velocity $u_{\theta}^{*}=r \Omega^{*}(r)$. In their paper, a piecewise linear function was chosen for $\Omega^{*}(r)$, and the transition zone was set equal to the cell height $h$.

In this paper, the forcing is expressed in terms of the vorticity function $\omega^{*}(r)=$ $2 \Omega^{*}(r)+r\left(\mathrm{~d} \Omega^{*} / \mathrm{d} r\right)$. Since the piecewise linear relation for $\Omega^{*}(r)$ employed by Chomaz et al. (1988) gives rise to discontinuous jumps in $\omega^{*}$ at the two sides of the shear layer (see figure 2), we have chosen a cubic interpolation for $\Omega^{*}(r)$. In addition, we have also used a $\tanh (r / \Delta r)$ relation for $\Omega^{*}$. The cubic interpolation has the advantage, aside from making $\omega^{*}(r)$ continuous, that it maintains a well-defined transition region. The tanh profile has continuous derivatives of all orders, but the width of the shear layer is not clearly bounded. In all the runs we present in this paper, the ratio of the normalizing length, $\Delta r$, in the tanh function to the shear layer width, $h$, is $\Delta r / h=0.4$. A comparison of the different shapes of $\Omega^{*}$ and $\omega^{*}$ in the transition region is shown in figure 2 .

Our numerical scheme has also been employed (Konijnenberg et al. 1998) in studies of the quasi-two-dimensional flow in a thin layer of fluid in a rotating, parabolic tank with an external shear. In this case, an additional linear term describing the variation of the Coriolis parameter, the so-called ' $\beta$-effect', is added to the left-hand side of (2.2). With this extra term, the flow can support inviscid, linear waves known as Rossby waves. In the case studied here, the inviscid limit of (2.2) is the Euler equation, which does not support any linear waves.

At the heart of both our asymptotic analysis and our numerical simulations is the approximation of the flow in an annular region in terms of a Fourier-Chebyshev 
series. All functions are expanded in the form

$$
\begin{aligned}
g(r, \theta, t) & =\sum_{n=-N / 2}^{N / 2-1} g_{n}(r, t) \mathrm{e}^{\mathrm{i} n \theta} \\
& =\sum_{n=-N / 2}^{N / 2-1} \sum_{m=0}^{M} g_{m n}(t) T_{m}(r) \mathrm{e}^{\mathrm{i} n \theta},
\end{aligned}
$$

where $M$ and $N$ are the orders of truncation and $T_{m}(r)$ (with normalized $r$ ) is the $m$ th-degree Chebyshev polynomial. In the following, the number of integer subindices will be used to indicate whether a field variable has $r, \theta$, and $t$ dependence (no subindex), $r$ and $t$ dependence (one subindex indicating the Fourier mode), or $t$ dependence only (two subindices indicating the Chebyshev and the Fourier mode, respectively). An important difference between our numerical schemes, used both in the asymptotic analysis and the direct simulations, and other traditional schemes is in the enforcement of the no-slip boundary conditions on the stream function $\psi$ as compatibility constraints on the vorticity $\omega$. A brief description of how both no-slip conditions and a 'free-slip' model are implemented through specific use of the spectral expansions in (2.4) is given in the next subsection.

\subsection{Boundary conditions}

The solution of the Poisson equation (2.3) requires boundary conditions at $r=r^{ \pm}$ for $\psi$. In the no-slip case, these boundary conditions are of both Dirichlet and Neumann type, indicating that the Poisson problem is over-determined. This apparent over-determinancy is resolved by enforcing solvability constraints on $\omega$. In our semiimplicit time-stepping scheme, these constraints are implemented directly as part of solving the Helmholtz equation for the vorticity field. This allows us to decouple the solution of the two linear problems for $\psi$ and $\omega$.

\subsubsection{No-slip boundary conditions}

The no-slip boundary condition on the fluid velocity

$$
\boldsymbol{u}\left(r^{ \pm}, \theta\right)=U^{ \pm}(t) \hat{\boldsymbol{\theta}}
$$

requires the stream function to satisfy the Neumann boundary conditions

$$
\left.\begin{array}{l}
\left.\frac{\partial \psi_{0}}{\partial r}\right|_{r^{ \pm}}=-U^{ \pm}(t), \\
\left.\frac{\partial \psi_{n}}{\partial r}\right|_{r^{ \pm}}=0 ; \quad n \neq 0,
\end{array}\right\}
$$

as well as the Dirichlet boundary conditions

$$
\left.\begin{array}{l}
\psi_{0}\left(r^{ \pm}\right)=F^{ \pm}(t), \\
\psi_{n}\left(r^{ \pm}\right)=0 ; \quad n \neq 0,
\end{array}\right\}
$$

where $F^{ \pm}(t)$ are arbitrary functions of time. The wall velocities, $U^{ \pm}(t)$, are also a function of time as we can spin-up (spin-down) the walls during the simulations.

The boundary conditions (2.6) and (2.7) will cause the Poisson equation (2.3) to be over-determined unless the $\omega_{n}(x)$ are restricted to ensure that both sets of conditions are fulfilled simultaneously. An accurate and efficient method for determining these 
'solvability constraints' is presented in Coutsias \& Lynov (1991) and Coutsias, Hesthaven \& Lynov (1996b), and a description of how this method is adapted to annular geometry is given in Coutsias et al. (1994). The result is a set of self-consistent no-slip boundary conditions

$$
\left.\begin{array}{rl}
\left.\frac{\partial \psi_{0}}{\partial r}\right|_{r=r^{ \pm}} & =-U^{ \pm}(t), \\
\psi_{n}\left(r^{ \pm}\right) & =0 ; \quad n \neq 0,
\end{array}\right\}
$$

together with

$$
\left.\begin{array}{rl}
\left.\frac{\partial \omega_{0}}{\partial r}\right|_{r=r^{ \pm}} & =-\frac{1}{v} \frac{\partial U^{ \pm}}{\partial t}, \\
\sum_{m=0}^{M} B_{n m}^{ \pm} \omega_{n m} & =0 ; \quad n \neq 0 .
\end{array}\right\}
$$

The constraint coefficients, $B_{n m}^{ \pm}$, can be determined accurately and efficiently by several equivalent methods. These coefficients are independent of the value of the viscosity, $v$, and they need only be calculated once for each different geometry, i.e. they only depend on the main radius, $a$, besides $M$ and $N$. Values of $B_{n m}^{ \pm}$obtained for high values of $M$ and/or $N$ can directly be used in calculations with lower resolution.

\subsubsection{Free-slip models}

As a direct consequence of the no-slip boundary conditions, narrow boundary layers containing high levels of vorticity are generated whenever the flow brings regions of vorticity close to the walls. The dynamical behaviour of the vorticity created in these boundary layers can have a very strong influence on the flow even at large distances from the walls (see, e.g., Coutsias \& Lynov 1991). Although no-slip boundary conditions are the most appropriate for most fluid systems, this is not the case in other physical systems, such as magnetized plasmas. In order to investigate the dynamical behaviour of forced annular shear flows in more generality without the influence of boundary-generated vorticity, the governing equations (2.2) and (2.3) are solved with boundary conditions modelling free-slip behaviour.

Introducing the azimuthal velocity component, $v$, the tangential stress on an element of the surface of a cylinder of radius $r$ is (see, e.g., Batchelor 1967, chapter 4.5)

$$
\sigma_{r \theta}=\mu\left(\frac{\partial v}{\partial r}-\frac{v}{r}\right) .
$$

From this expression it is clear that a 'stress-free' situation occurs when

$$
\frac{\partial v}{\partial r}=\frac{v}{r} .
$$

Since the radial velocity component is zero at the cylindrical walls, the stress-free condition can be formulated for vorticity as

$$
\omega=\frac{2 v}{r} \quad \text { at } r=r^{ \pm} .
$$

Translating to stream function, the boundary conditions for the Poisson equation are

$$
\left.\begin{array}{rl}
\psi_{n}\left(r^{ \pm}\right) & =0 ; \quad n \neq 0, \\
\left.\frac{\partial \psi_{0}}{\partial r}\right|_{r=r^{ \pm}} & =-\frac{r^{ \pm} \omega_{0}\left(r^{ \pm}\right)}{2} .
\end{array}\right\}
$$


In the asymptotic analysis described in the next section, the linear problem is expressed as an Orr-Sommerfeld problem in $\psi$ and the stress-free condition (2.10) is used as a 'free-slip' model.

In the full numerical solution of (2.2) and (2.3), (2.12) is used as boundary condition for the Poisson equation. The semi-implicit time-stepping scheme also requires boundary conditions on $\omega$ for the solution of the Helmholtz equation. For this purpose (2.10) is not well-suited since it uses information about $v$ from a previous time step to advance the vorticity. This introduces a time-splitting error of $O(\Delta t)$ into the code, compromising the $O\left(\Delta t^{3}\right)$ accuracy of the scheme. So, rather than using the stress-free condition (2.10) in the simulations 'free-slip' conditions are modelled by requiring zero radial gradient of the vorticity at the boundaries,

$$
\left.\frac{\partial \omega}{\partial r}\right|_{r=r^{ \pm}}=0 .
$$

Whether this boundary condition is more or less appropriate than (2.10) as an idealized model for a given fluid experiment is not easily determined, since they both may be impossible to realize. However, (2.13) is a mathematically legitimate boundary condition to the Helmholtz equation. It also is a reasonable boundary condition when modelling the dynamics of electrostatic fluctuations in the outer annular part of a cylindrical plasma confined in a magnetic field. In this case, the dynamical equations take the same form as in the fluid case, but the variable $\omega$ represents the charge density rather than the vorticity (see, e.g., Pécseli et al. 1992). As will become clear in the following, the 'free-slip' model (2.13) works precisely as intended since it prevents strong boundary layers being formed without affecting the flow away from the walls.

\subsection{Temporal evolution of global quantities}

The temporal evolution of the total energy, $E \equiv \frac{1}{2} \int_{D}|\boldsymbol{u}|^{2} \mathrm{~d} A$, the total enstrophy, $W \equiv \int_{D} \omega^{2} \mathrm{~d} A$, and the total angular momentum, $L \equiv \int_{D} \boldsymbol{u} \times \boldsymbol{r} \cdot \hat{\boldsymbol{e}}_{z} \mathrm{~d} A$, can be obtained from the Navier-Stokes equations. The results are

$$
\begin{gathered}
\frac{\mathrm{d} E}{\mathrm{~d} t}=-v W(t)+v U^{+} r^{+} \int_{0}^{2 \pi} \omega\left(r^{+}, \theta\right) \mathrm{d} \theta \\
-v U^{-} r^{-} \int_{0}^{2 \pi} \omega\left(r^{-}, \theta\right) \mathrm{d} \theta+\int_{D} \boldsymbol{u} \cdot \boldsymbol{F} \mathrm{d} A, \\
\frac{\mathrm{d} W}{\mathrm{~d} t}=2 v \int_{\partial D} \omega \nabla \omega \cdot \hat{\boldsymbol{n}} \mathrm{d} s-2 v \int_{D}(\nabla \omega)^{2} \mathrm{~d} A+2 \int_{D} \omega(\nabla \times \boldsymbol{F}) \cdot \hat{\boldsymbol{e}}_{z} \mathrm{~d} A,
\end{gathered}
$$

and

$$
\begin{aligned}
\frac{\mathrm{d} L}{\mathrm{~d} t}=2 \pi v\left(-r^{+2} \omega_{0}\left(r^{+}\right)+r^{-2} \omega_{0}\left(r^{-}\right)\right. & \left.+2 r^{+} U^{+}-2 r^{-} U^{-}\right) \\
& -\frac{16 \pi v}{h^{2}} \int_{r^{-}}^{r^{+}} r^{2}\left[u_{\theta}^{*}(r)-u_{\theta}(r)\right] \mathrm{d} r,
\end{aligned}
$$

where $U^{ \pm}$are the flow velocities at the boundaries $r^{ \pm}, \hat{\boldsymbol{n}}$ is the outgoing normal to the boundary, the subindex 0 denotes the zeroth azimuthal Fourier mode, and $\boldsymbol{F}$ is the Ekman forcing expressed as velocity

$$
\boldsymbol{F}=\frac{8 v}{h^{2}}\left(\boldsymbol{u}^{*}-\boldsymbol{u}\right) .
$$


It is seen that the only terms which can generate energy, enstrophy and angular momentum are either boundary terms or terms containing the forcing velocity $\boldsymbol{u}^{*}=$ $u_{\theta}^{*} \hat{e}_{\theta}$. As described in $\S 5,(2.14)-(2.16)$ are used as accuracy checks of the numerical solution.

\section{Asymptotic analysis}

Before describing details of the perturbation hierarchy in the next two subsections, we will give a unified introduction to our linear and weakly nonlinear analysis. It will be shown from the linear analysis that the linearized operator about the radially symmetric state is of Fredholm type. Thus, we can apply the Fredholm alternative theorem to suppress secular terms in the variational equations. In the analysis, we are guided from the results of the direct simulations (but also as a direct consequence of the form of the problem). Thus, we anticipate the existence of a critical Reynolds number, $R e_{c}$ (with a corresponding critical viscosity, $v_{c}$ ) below which the flow is stable. We therefore employ the small parameter $\varepsilon=\left(1 / v-1 / v_{c}\right)^{1 / 2}$ (note that by fixing length and wall velocity scales we can only affect the Reynolds number through varying the viscosity). Thus, $v=1 /\left(R e_{c}+\varepsilon^{2}\right)=v_{c}\left(1-\varepsilon^{2} v_{c}+O\left(\varepsilon^{4}\right)\right)$.

We introduce the slow time scale $\tau=\varepsilon t$ and we expand the flow fields in the form

$$
g(r, \theta, t)=g_{0}(r)+\sum_{k=1}^{3} \varepsilon^{k} g_{k}(r, \theta, t, \tau)+O\left(\varepsilon^{4}\right) ;
$$

here $g$ stands for either $\omega$ or $\psi$. The slow time scale is suggested, as usual, by the dependence of the unstable eigenvalues on $R e$ in the vicinity of $R e=R e_{c}$. The analysis of the $O\left(\varepsilon^{0}\right)$ and $O\left(\varepsilon^{1}\right)$ problems gives the basic linearized stability picture. It reveals that the linearized problem has a pair of eigenvalues crossing the imaginary axis for some critical value of viscosity $\left(R e_{c}\right)$, so that a conjugate pair of modes becomes neutrally stable at $R e=R e_{c}$. For $R e$ slightly above $R e_{c}$ (i.e. for small $\varepsilon$ ), the weakly nonlinear analysis proceeds by considering the evolution of a perturbation of the form

$$
\left(\begin{array}{l}
\omega_{1}(r, \theta, t) \\
\psi_{1}(r, \theta, t)
\end{array}\right) \approx A \mathrm{e}^{\lambda t+\mathrm{i} n \theta}\left(\begin{array}{l}
\Omega_{1}(r) \\
\Psi_{1}(r)
\end{array}\right)+\text { C.C. }
$$

For each azimuthal mode number, $n$, the value $R e=R e_{s}$ giving marginal stability, i.e. $\operatorname{Re}(\lambda)=0$, is found. The critical Reynolds number is determined by $\operatorname{Re}_{c}=\min _{n}\left(R e_{s}\right)$, which also specifies the first unstable mode number $n=n_{c}$.

To $O\left(\varepsilon^{2}\right)$ the perturbation equations produce non-resonant second (and zero) harmonic corrections, but to $O\left(\varepsilon^{3}\right)$ the interaction of these harmonics with the fundamental produces resonant (secular) terms. Suppressing these leads to a Landau equation for the slowly varying amplitude $A(\tau)$ (Drazin \& Reid 1991).

\subsection{The linear analysis}

We analyse here the first two equations in the perturbation hierarchy resulting from substituting (3.1) into (2.2) and (2.3). The $O\left(\varepsilon^{0}\right)$ equations are

$$
\begin{gathered}
\Delta \psi_{0}=-\omega_{0}, \\
\Delta \omega_{0}+\frac{8}{h^{2}}\left(\omega^{*}-\omega_{0}\right)=0,
\end{gathered}
$$

together with either the free-slip boundary conditions (2.10) and (2.12) or the noslip conditions (2.8)-(2.9) with stationary $U^{ \pm}$. It is clear that the steady state is 


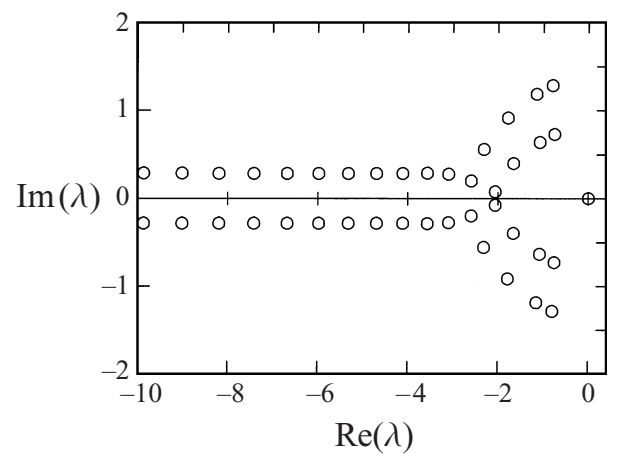

FigURE 3. Typical spectrum $\left(a=3.0, U^{-}=0.63, U^{+}=-0.74, \Gamma=6.5, R e=86.2\right.$, cubic profile for $\Omega^{*}$, and no-slip boundary conditions). The inner and outer wall velocities were chosen in order to select the reference frame of the neutral mode. Consequently, a double, non-degenerate eigenvalue is crossing the imaginary axis through the origin. Only values with real parts greater than -10 are shown (here $M=200$ ).

independent of viscosity. Given the form of $\omega^{*}$, the above problem must be solved numerically. This is done here through a highly accurate Chebyshev spectral scheme.

For the analysis of the $O\left(\varepsilon^{1}\right)$ problem, we separate variables and restrict our attention to eigenmodes of specific azimuthal symmetry of order $n$, assuming the form (3.2). Writing $\Delta_{n}$ for $\partial_{r}^{2}+(1 / r) \partial_{r}-\left(n^{2} / r^{2}\right)$, we obtain

$$
\begin{gathered}
\Delta_{n} \Psi_{1}=-\Omega_{1}, \\
v \Delta_{n} \Omega_{1}-\frac{8 v}{h^{2}} \Omega_{1}=\frac{\mathrm{i} n}{r}\left(\Psi_{1} \partial_{r} \omega_{0}-\Omega_{1} \partial_{r} \psi_{0}\right)+\lambda \Omega_{1},
\end{gathered}
$$

with either free-slip or no-slip boundary conditions on $\Psi_{1}$ and $\Omega_{1}$, both with homogeneous boundary conditions. The problem is solved numerically in the form

$$
v \Delta_{n}^{2} \Psi_{1}+\frac{\mathrm{i} n}{r}\left(\Psi_{1} \partial_{r} \omega_{0}+\Delta_{n} \Psi_{1} \partial_{r} \Psi_{0}\right)=\left(\lambda+\frac{8 v}{h^{2}}\right) \Delta_{n} \Psi_{1} .
$$

The calculation is carried out for arbitrary $v$ (and $R e$ ) and a secant iteration is employed to estimate the critical value of viscosity, $v_{c}$, at which a complex conjugate eigenpair first crosses the imaginary axis. Actually, we solve the problems for $\pm n$ simultaneously. Since we are using the SLATEC qz-factorization algorithm, written for the real generalized eigenproblem $A x=\lambda B x$, we convert the problem to one for real matrices of twice the size. Again, our computation involves expansion in Chebyshev polynomials. The boundary conditions are included as Lanczos $\tau$-conditions in the truncated matrix operators. For any given truncation $M$, the analysis results in a spectrum of (generally) complex eigenvalues. As the truncation is increased, some of the computed eigenvalues will stay unchanged to high precision; these are the eigenvalues whose eigenmodes were adequately resolved at truncation $M$. In contrast, the eigenvalues of poorly resolved modes are quite sensitive to truncation. Several studies were carried out (Bergeron 1993) of the behavior of the spectrum of the linearized problem under small perturbations of the coefficient matrices. The resulting pseudo-spectrum proved remarkably robust for the well-resolved regime.

Our studies show that the spectrum nearest to the origin is resolved first (see figure 3), and as higher modes are added to the computation the new eigenvalues 'stabilize' further and further away along the negative real axis. This is consistent with, among other things, the well-posedness and Fredholm character of the variational 


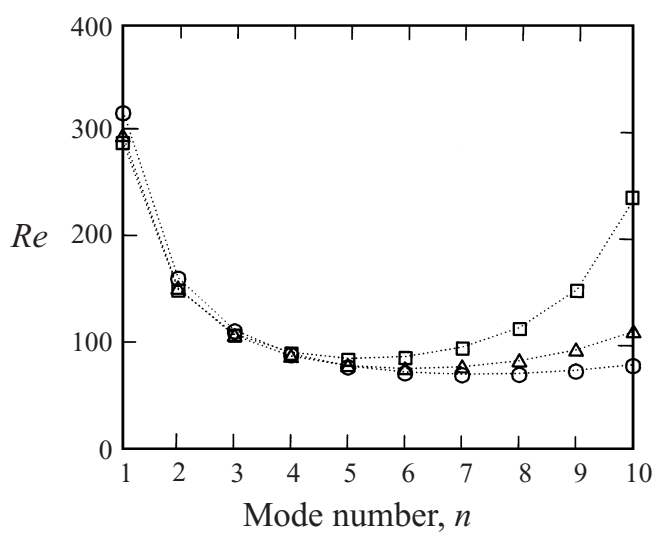

FIGURE 4. Marginal stability curves for $a=3.0, \Gamma=6.5$ (squares), 8 (triangles), 10 (circles), cubic profile for $\Omega^{*}$, and no-slip boundary conditions. The symbols show results from linear analysis for the value of the marginally stable Reynolds number, $R e_{s}$, at which a given mode number, $n$, becomes unstable. The dotted lines are only drawn to guide the eye. The critical values $R e_{c}$ and $n_{c}$ for a given value of $\Gamma$ are determined by the lowest value of $\operatorname{Re}_{s}(n)$.

operator near the circular state (see, e.g., Stakgold 1975). The eigenvalues in the vicinity of the origin can thus be computed with a small $(\approx 50)$ number of Chebyshev modes so that they remain unchanged to $8-9$ decimal places as the truncation is further increased. This allows us to interpolate accurately the value of the Reynolds number, $R e_{c}$, at which a pair crosses the imaginary axis at $\pm \beta$. We find that the pair crossing the imaginary axis is isolated. The form of the operators implies that a change to a rotating frame with relative angular velocity $\Theta$ will shift the spectrum so that each conjugate pair will move parallel to the imaginary axis by $\pm n \Theta$ (i.e. the various eigenmodes are damped standing waves each in a different rotating frame). If we change the angular velocity of the background frame by the amount $\beta / n$ through an appropriate modification of the boundary conditions of the $O\left(\varepsilon^{0}\right)$ problem, we will find a double eigenvalue crossing through the origin. Our subsequent analysis is carried out in this frame.

In order to determine which mode becomes unstable first, this analysis must be repeated for several values of $n$. The marginal stability curves for several $n$ and for different values of the aspect ratio $\Gamma$ are shown in figure 4 . The resulting prediction for the first unstable mode as a function of aspect ratio agrees with the empirical rule

$$
n_{c} / \Gamma \approx 0.77 \pm 0.03
$$

deduced by Rabaud \& Couder (1983). We note that $R e_{c}$ depends also on the value of $\Gamma$.

The vorticity fields corresponding to the eigenfunctions of a marginally stable state with $a=3, \Gamma=6.5$, cubic profile for $\Omega^{*}, R e=89.32$, and both free-slip and no-slip boundary conditions are shown in figures $5(a)$ and $5(d)$, respectively. We note that the eigenfunctions for these two cases are very similar except close to the walls.

\subsection{The Landau equation}

The $O\left(\varepsilon^{2}\right)$ problem is solved in the form

$$
\left(\begin{array}{cc}
r^{2} \Delta_{n} & r^{2} I \\
-r \partial_{r} \omega_{0} \partial_{\theta} & v r^{2} \Delta_{n}-\frac{8 v r^{2}}{h^{2}} I+r \partial_{r} \psi_{0} \partial_{\theta}
\end{array}\right)\left(\begin{array}{c}
\psi_{2} \\
\omega_{2}
\end{array}\right) \equiv L\left(\begin{array}{c}
\psi_{2} \\
\omega_{2}
\end{array}\right)=\left(\begin{array}{c}
0 \\
r^{2} J\left(\Omega_{1}, \Psi_{1}\right)
\end{array}\right)
$$


(a)

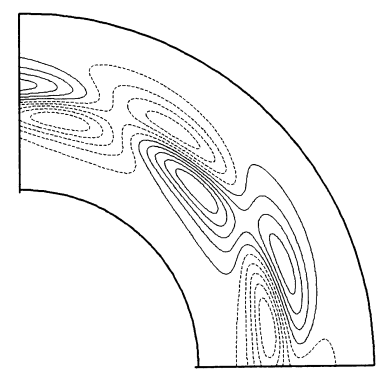

$(d)$

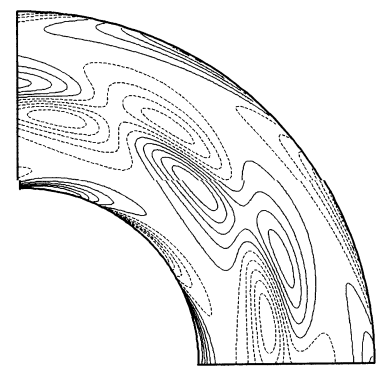

(b)

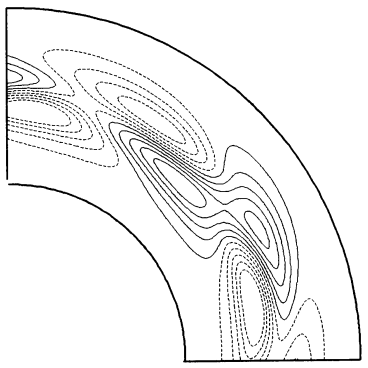

(e)

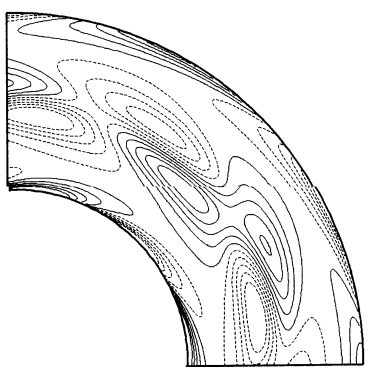

(c)

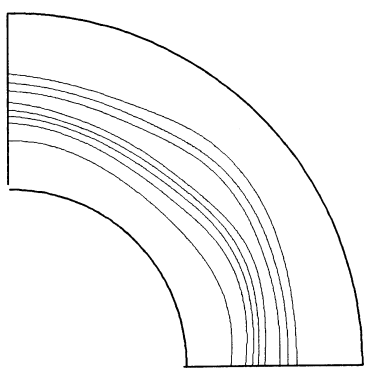

$(f)$

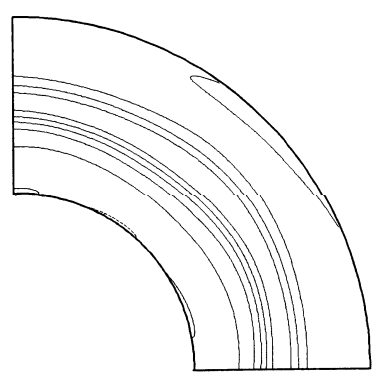

FIGURE 5. Vorticity fields of a one quarter section of the first unstable mode for $a=3, \Gamma=6.5$, and cubic profile for $\Omega^{*}$. Dotted lines indicate negative values and solid lines positive values. $(a-c)$ Free-slip boundary conditions; $(d-f)$ no-slip conditions. $(a, d)$ Eigenfunctions for the marginally stable case from linear analysis; $(b, e)$ saturated perturbation from full numerical simulation at $R e=89.32 ;(c, f)$ total saturated field from the same numerical simulation as in $(b, e)$. In all the figures, the amplitude has been normalized to 1 .

with similar homogeneous boundary conditions as for the $O\left(\varepsilon^{1}\right)$ system. Here we have

$$
r^{2} J\left(\Omega_{1}, \Psi_{1}\right)=\mathrm{i} n r A^{2}\left(\partial_{r} \Omega_{1} \Psi_{1}-\partial_{r} \Psi_{1} \Omega_{1}\right) \mathrm{e}^{2 \mathrm{i} n \theta}+\mathrm{i} n r A \bar{A}\left(\partial_{r} \bar{\Omega}_{1} \Psi_{1}-\partial_{r} \bar{\Psi}_{1} \Omega_{1}\right)+\text { C.C. }
$$

As we found in the eigenvalue analysis, the crossing eigenvalues are isolated and the linearized operator is Fredholm. So, in the proper frame of the neutral mode, we have a double eigenvalue crossing through zero, and the linearized operator has two null modes. Thus, by the Fredholm alternative theorem (Stakgold 1975), the non-homogeneous problem $L u=f$ will have a solution only if the right-hand side, $f$, is orthogonal to the adjoint null modes. Here, this orthogonality is automatic through the $\theta$-dependence of the right-hand side: it is only terms of the form $\exp ( \pm \mathrm{i} n \theta)$ that are resonant with a neutral mode. In the $O\left(\varepsilon^{2}\right)$ calculation no resonant terms appear, so we proceed with separation of variables and we find, by numerical solution of the resulting ordinary differential equations in $r$ (with $g$ standing as before for either $\omega$ or $\psi)$ :

$$
g_{2}=|A|^{2} G_{20}(r)+A^{2} G_{22}(r) \mathrm{e}^{2 \mathrm{i} n \theta}+\bar{A}^{2} \bar{G}_{22}(r) \mathrm{e}^{-2 \mathrm{in} \theta} .
$$

Finally, the $O\left(\varepsilon^{3}\right)$ problem gives

$$
L\left(\begin{array}{c}
\psi_{3} \\
\omega_{3}
\end{array}\right)-\left(\begin{array}{c}
0 \\
v_{c}\left(J\left(\omega_{0}, \Psi_{1}\right)+J\left(\Omega_{1}, \psi_{0}\right)\right)+\partial_{\tau} \Omega_{1}
\end{array}\right)=\left(\begin{array}{c}
0 \\
r^{2}\left(J\left(\Omega_{1}, \psi_{2}\right)+J\left(\omega_{2}, \Psi_{1}\right)\right)
\end{array}\right)
$$

(the additional term on the left-hand side results from the $O\left(\varepsilon^{2}\right)$ perturbation in the Reynolds number). 
Thus, we find that the right-hand side contains resonant terms. Since non-resonant forcing will only produce a bounded response to this order, we need to concentrate on the resonant terms only. Imposing orthogonality with the null solutions of the adjoint problem leads to the Landau equation

$$
\frac{\mathrm{d} A}{\mathrm{~d} \tau}=\alpha A+\xi|A|^{2} A
$$

describing the saturation behaviour of the neutral mode. The details of this calculation can be found in Bergeron (1993).

The numerical solution of the variational equations for the $O\left(\varepsilon^{2}\right)$ and $O\left(\varepsilon^{3}\right)$ expansions has proven to be very difficult. It has not yet been possible to achieve the high accuracy obtained in the numerical solution of the linear problem reported in the previous subsection. The difficulties are related to the ill-conditioning of the Chebyshev differentiation matrix for higher-order derivatives and at high truncations numbers (see, e.g., Don \& Solomonoff 1995). We have derived both post-conditioning (Coutsias et al. 1995) and pre-conditioning (Coutsias et al. 1996a) spectral methods to solve similar problems efficiently maintaining high accuracy. These methods are at present being applied to the solution of the $O\left(\varepsilon^{2}\right)$ and $O\left(\varepsilon^{3}\right)$ problems and the results will be reported elsewhere.

\section{Numerical scheme}

Inserting the Fourier-Chebyshev expansion (2.4) into the dynamical flow equations (2.2) and (2.3) yields the equations describing the temporal evolution of the spectral expansion coefficients $\omega_{m n}(t)$. For the time-stepping of these equations, we have employed the third-order 'Stiffly-Stable' scheme described by Karniadakis, Israeli \& Orszag (1991). This is a mixed explicit/implicit time-integration method in which the linear terms in $\omega_{m n}$ contained on the right-hand side of (2.2) are treated implicitly, while the nonlinear term described by the Jacobian on the left-hand side of (2.2) is treated explicitly.

The implicit terms in the time-integration give rise to a Helmholtz equation for $\omega_{m n}$. Thus, at each time step a Poisson equation for $\psi_{m n}$, originating from (2.3), and a Helmholtz equation for $\omega_{m n}$ have to be solved. The boundary conditions for these equations are described by either the no-slip conditions (2.8) and (2.9) or the free-slip conditions (2.12) and (2.13). Suitable boundary conditions are included via the 'taumethod' (see, e.g., Gottlieb \& Orszag 1977) in which the two rows in the matrices for the Poisson and the Helmholtz equations describing the modes with the two highest mode numbers are substituted by the boundary conditions at $r=r^{+}$and $r=r^{-}$. Direct numerical solution of each of these linear systems requires $O\left(M^{3} N\right)$ operations, which is unacceptable, and would not be very accurate due to ill-conditioning of the matrices. However, Chebyshev recursion relations (see, e.g., Gottlieb \& Orszag 1977) allow the transformation of the matrices into a triangularized, penta-diagonal form, except for the two rows containing the boundary conditions. Subsequent Gauss elimination produces well-conditioned, lower triangular banded matrices ready for solution by direct forward substitution. These row operations involved in the Gauss elimination are only performed initially. The actual solution of the Helmholtz and Poisson equation by this scheme requires only $O(M N)$ operations and yields spectrally accurate results even for high values of $M$ and $N$. Further details on the solution of the Helmholtz and Poisson equations are given by Nielsen (1993) and Bergeron (1993). 
In the explicit calculation of the nonlinear term described by the Jacobian, the products are calculated in point space and the result is fully de-aliased using the standard 2/3 truncation scheme (see, e.g., Coutsias et al. 1989). Due to the high efficiency of our Poisson and Helmholtz solvers, $75-80 \%$ of the CPU time in the numerical simulation is spent in the FFT routines involved in the transformations between mode space and configuration space for the calculation of the Jacobian. However, these FFT routines are usually highly optimized library routines specific for the particular computer on which the code is run. The present simulations are performed on a Cray C92A, located at UNI-C, Lyngby, Denmark, and for a resolution of 256 Chebyshev polynomials and 256 Fourier modes we were able to perform 1000 time steps in $90 \mathrm{~s}$.

\section{Numerical results}

As mentioned above, the dynamical evolution of the annular shear layer depends on several factors besides the Reynolds number, $R e$, and the shear layer aspect ratio, $\Gamma$. These additional factors are the wall boundary conditions (no-slip or free-slip), the location of the shear layer relative to the walls (in our case the shear layer is always centred midway between the walls so that the distances are determined through the cell aspect ratio, $\left.a=\left(r^{-}+r^{+}\right) /\left(r^{-}-r^{+}\right)\right)$, the precise shape of the shear layer profile, and the ramp-up rate, $\mathrm{d} R e / \mathrm{d} t$, during spin-up and spin-down experiments. Furthermore, the initial condition for the vorticity field influences the precise values of the vorticity at a given point at a given time during the evolution. In all the numerical runs presented here, the initial conditions were chosen as a superposition of the basic flow, determined by numerical solution of the zero-order equations (3.3) and (3.4), and some small-amplitude perturbation. We have found that the dynamical behaviour of the forced/damped shear flow is not very sensitive to changes in the perturbing field. Thus, a large variety of different initial conditions lead to geometrically similar vorticity fields which only differ by a constant azimuthal phase. It is the existence of these well-defined attractors for the nonlinear flow field that gives the following investigations general validity.

It is not possible to cover the entire function space spanned by all the external parameters through numerical simulations. However, in this section we will try to illustrate the influence of several of these parameters through different test cases. In $\S 5.1$, numerical results obtained near the first bifurcation from an axisymmetrical state are presented. Here the effects of boundary conditions are investigated and comparisons are performed with the theoretical results from the asymptotic analysis in $\S 3$ as well as with the experimental results of Rabaud \& Couder (1983) and Chomaz et al. (1988). In $\S 5.2$, numerical results for spin-up and spin-down experiments are shown and the effects of the change of spin rate and external noise are described. The runs in $\S 5.2$ are carried out for a different shear profile and for different values of $\Gamma$ and $a$ compared to those in $\S 5.1$ in order to demonstrate the effect of these parameters. Finally, in $\S 5.3$ some examples are shown of the dynamical behaviour at large Reynolds numbers and the effects of different boundary conditions are investigated.

\subsection{Near linear results}

In this subsection comparisons are performed between the results from the full numerical simulations and from the asymptotic analysis. The parameters in the runs were chosen so that comparison with previously published experimental results could 


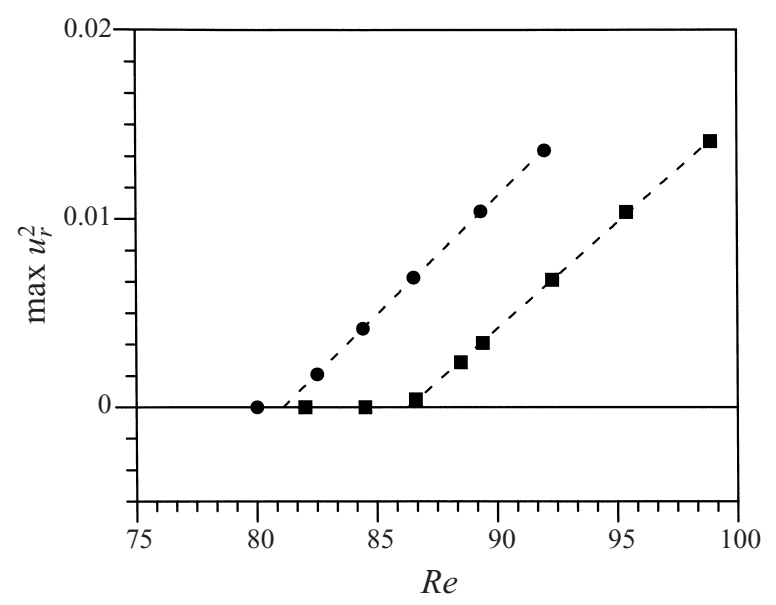

FiguRE 6. Supercritical bifurcation near the critical Reynolds number for $a=3, \Gamma=6.5$, and cubic profile for $\Omega^{*}$. The squares mark the values of the saturated amplitude of the square of the maximal radial velocity, $\max u_{r}^{2}$, for different values of the Reynolds number $R e$ and no-slip boundary conditions. The circles show similar results for free-slip boundary conditions. The dashed lines indicate linear, least-squares fits to the points with non-axisymmetric flows.

also be carried out. All the runs were performed with $a=3, \Gamma=6.5, M=N=256$, and with cubic interpolation for the shear forcing as described in $\S 2$. Both no-slip and free-slip boundary conditions were investigated.

In order to check the accuracy of our numerical solution, we have included a number of error estimates in the code. Let $F$ denote any of the global quantities energy, $E$, enstrophy, $W$, or angular momentum, $L$. At fixed time intervals, typically for every 100 time steps, we calculate a time-centred, fourth-order-accurate value of the time derivative of $F$, denoted $(\mathrm{d} F / \mathrm{d} t)_{\text {num }}$, determined by evaluation of $F$ at five sequential time steps. By employing a fourth-order estimation, we ensure consistency in approximation level with the third-order-accurate, stiffly-stable time integration scheme. This numerical time derivative is compared to the theoretical value $(\mathrm{d} F / \mathrm{d} t)_{\text {theor }}$ evaluated from the instantaneous fields entering the right-hand sides of the respective evolution equations of $F$ presented in $\S 2.2$. As an error estimate, $\delta F$, we employ the relative difference per time unit of these two time derivatives values, so

$$
\delta F(t)=\left|\frac{(\mathrm{d} F / \mathrm{d} t)_{\text {num }}-(\mathrm{d} F / \mathrm{d} t)_{\text {theor }}}{F(t)}\right| .
$$

In all the runs in this subsection, the accuracy checks gave $\delta E \leqslant 5 \times 10^{-7}, \delta W \leqslant$ $2 \times 10^{-6}$ and $\delta L \leqslant 10^{-10}$.

Figure 6 shows the numerical results for the nonlinear saturation of the perturbation near the first bifurcation from the axisymmetric state. The symbols indicate the saturated level of the maximal radial velocity squared, $\max u_{r}^{2}$, for various values of $R e$. The squares and circles indicate results for no-slip and free-slip boundary conditions, respectively. The calculations were initialized by exciting the first 20 azimuthal modes with random phases and low amplitudes. For Re less than a welldefined critical value, $R e_{c}$, all the azimuthal modes die out (down to zero with machine accuracy) and an axisymmetric state is restored. For $R e$ values not too far above $R e_{c}$, an $n=5$ mode is excited in agreement with the linear analysis for $\Gamma=6.5$ and $a=3$. After a transient period, during which the stable modes die out, the $n=5$ 
perturbation grows to a finite amplitude and saturates. A linear, least-squares fit to the results above $R e_{c}$ is indicated by dashed lines. It is clearly seen that the saturated amplitudes of $u_{r}$ grow as $\left(R e-R e_{c}\right)^{1 / 2}$ characteristic of the supercritical bifurcation assumed in the asymptotic analysis in $\S 3$.

The intersections of the dashed lines in figure 6 with the $R e$-axis give good estimates of $R e_{c}$. The values for $R e_{c}$ found this way are $81.1 \pm 0.1$ (free-slip) and $R e_{c}=86.3 \pm 0.1$ (no-slip). These values are in good agreement with the results from the asymptotic analysis, which gives $R e_{c}=80.6$ and $R e_{c}=86.18$ for the free-slip and no-slip cases, respectively. Experimentally, Rabaud \& Couder (1983) found $R e_{c}=85 \pm 5$, while Chomaz et al. (1998) reported $R e_{c}=80 \pm 2$. When comparing the analytical and numerical results with the experimental results we believe that the no-slip boundary conditions are the most appropriate. In both experimental papers the values for $R e_{c}$ are found to be fairly constant over a broad range of $\Gamma$-values, including the value $\Gamma=6.5$ used in this section. We see that our numerical and asymptotic results are in reasonable agreement with the experiment, although we find a clear dependence of $R e_{c}$ on $\Gamma$. Furthermore, by changing the shear forcing profile in the numerical runs and the asymptotic analysis we can vary the numerical value of $R e_{c}$ for both types of boundary conditions. This can easily be obtained with the tanh profile, where the length scale parameter in the tanh function can be chosen freely over a broad range of values around the shear layer width, $h$, so we could achieve an even better agreement with the experimental values by optimizing the shear profile. We note that in the numerical simulations of Chomaz et al. (1988), the critical Reynolds number was found to be $R e_{c} \approx 60$ for $\Gamma=6.5$ (see their figure 9). However, as also mentioned in Chomaz et al. (1988), the large discrepancy with the experiment was not surprising, since their numerical simulations were based on a fairly crude numerical model.

In figure 5 , the saturated vorticity fields for both free-slip and no-slip boundary conditions at $R e=89.32$ are shown. Figures $5(c)$ and $5(f)$ show the total vorticity field at saturation for the free-slip and no-slip cases, respectively, while figures $5(b)$ and $5(e)$ show the corresponding perturbations obtained by subtracting the zero-order fields. The good agreement between the linear results and the results from the full numerical simulation is obvious. We note that the 'free-slip' boundary conditions used in the asymptotic analysis and in the full simulation are based on two different models, as described in $\S 2.1 .2$. Nevertheless, figure 5 shows that the two different models, at least in this case, give virtually indistinguishable results. We have also calculated the saturated perturbation for the other $R e$ values represented in figure 6 and found that the geometry of the fields corresponding to the two different boundary conditions only changed very slightly for $R e_{c}<R e<100$.

Figure 7 shows the evolution of energy and enstrophy during transition from almost axisymmetric initial conditions to saturation at $R e=89.32$ for no-slip boundary conditions. Figure 7(a) shows the temporal evolution of the normalized total energy, $E$, and the normalized total enstrophy, $W$ and we clearly see that both quantities are lower in the saturated state with mode 5 than in the axisymmetric state. This drop in energy and enstrophy increases as $R e$ is increased. Figure $7(b)$ shows the evolution of the energy content on a logarithmic scale of the 10 lowest azimuthal modes (a similar plot for the enstrophy demonstrates the same features). As mentioned above, the first 20 modes were initially excited with random phases and low amplitudes. In figure $7(b)$ we see that both mode 5 and mode 6 start growing exponentially in accordance with linear analysis. Up to $t=300$ we note that the flow evolves linearly, and then nonlinear effects set in. Around $t=400$, mode 5 begins to dominate the flow and mode 6 starts to die out. Meanwhile the higher harmonics to mode 5 have 

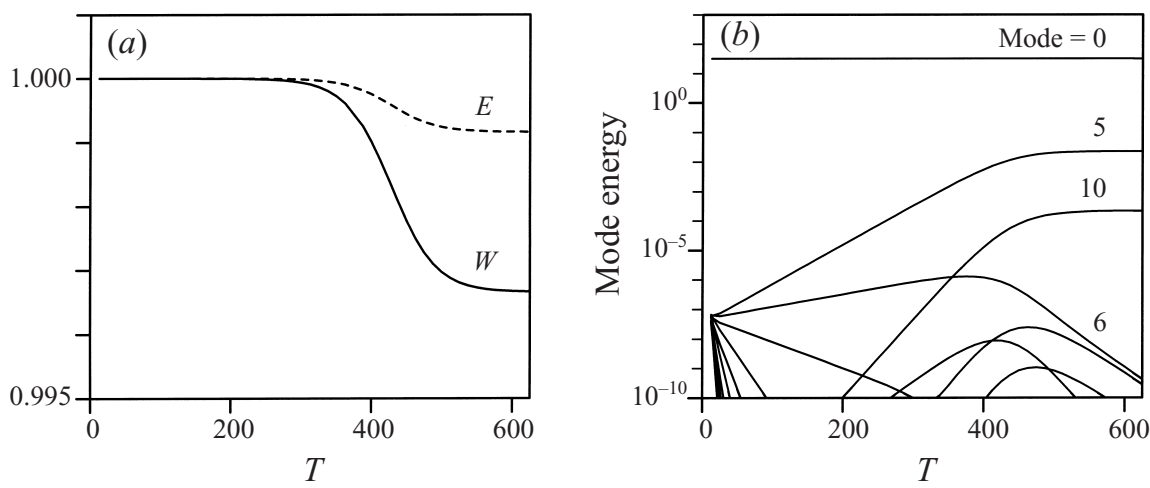

FigURE 7. Evolution of energy and enstrophy during transition at $\operatorname{Re}=89.32$ for $a=3, \Gamma=6.5$, cubic profile for $\Omega^{*}$, and no-slip boundary conditions. (a) Temporal behaviour of normalized total energy, $E$, and normalized total enstrophy, $W .(b)$ Temporal behaviour of the energy content in the 10 lowest azimuthal modes.

\begin{tabular}{lccccc}
\hline Boundary conditions & Analytical $\alpha_{r}$ & $\alpha_{r}$ & $\alpha_{i}$ & $\tilde{\xi}_{r}$ & $\tilde{\xi}_{i}$ \\
Free-slip & 0.045 & 0.04200 & 0.06451 & -0.11325 & 0.007389 \\
No-slip & 0.0158 & 0.01545 & 0.06386 & -0.04322 & 0.003078
\end{tabular}

TABLE 1. Landau coefficients for the runs shown in figure 8.

been excited (in the figure only mode 10 is shown). At the end of the run, the system is very close to the asymptotic state since modes 5 and 10 have levelled off and the other modes, though still decaying, have much lower amplitudes than the saturated modes. We note that the energy content in mode 0 drops during the run as expected from figure $7(a)$, but that this drop is not visible on the logarithmic scale used in figure $7(b)$.

In order to determine the value of the coefficients in the Landau equation (3.8) from the numerical solutions, some additional runs were performed. In these runs, the initial perturbations were based on the saturated vorticity fields such as the one shown in figures $5(b)$ and $5(e)$. From such a field, the Chebyshev expansion coefficients, $m$, for Fourier mode $n=5$ are extracted, multiplied by $10^{-3}$ and used as initial perturbation to the basic flow. The results from the numerical simulations give easy access to information on the temporal evolution of the amplitude and phase of this perturbation. To facilitate comparison of the evolution of these quantities with the Landau equation (3.8), we introduce the two real variables $\mathscr{A}(\tau)$ and $\phi(\tau)$ defined by $A(\tau)=\mathscr{A}(\tau) \exp [\mathrm{i} \phi(\tau)]$. Furthermore, we introduce $\alpha=\alpha_{r}+\mathrm{i} \alpha_{i}, \xi=\xi_{r}+\mathrm{i} \xi_{i}$, and the amplitude of the perturbed vorticity field $\hat{\omega}=2 \mathscr{A}$. Substituting these variables into (3.8) we obtain

$$
\frac{\mathrm{d} \hat{\omega}}{\mathrm{d} t}=\alpha_{r} \hat{\omega}+\tilde{\xi}_{r} \hat{\omega}^{3}
$$

and

$$
\frac{\mathrm{d} \phi}{\mathrm{d} t}=\alpha_{i}+\tilde{\xi}_{i} \hat{\omega}^{2}
$$

Here, we have introduced the scaled constants $\tilde{\xi}_{r}=\xi_{r} / 4$, and $\tilde{\xi}_{i}=\xi_{i} / 4$. 

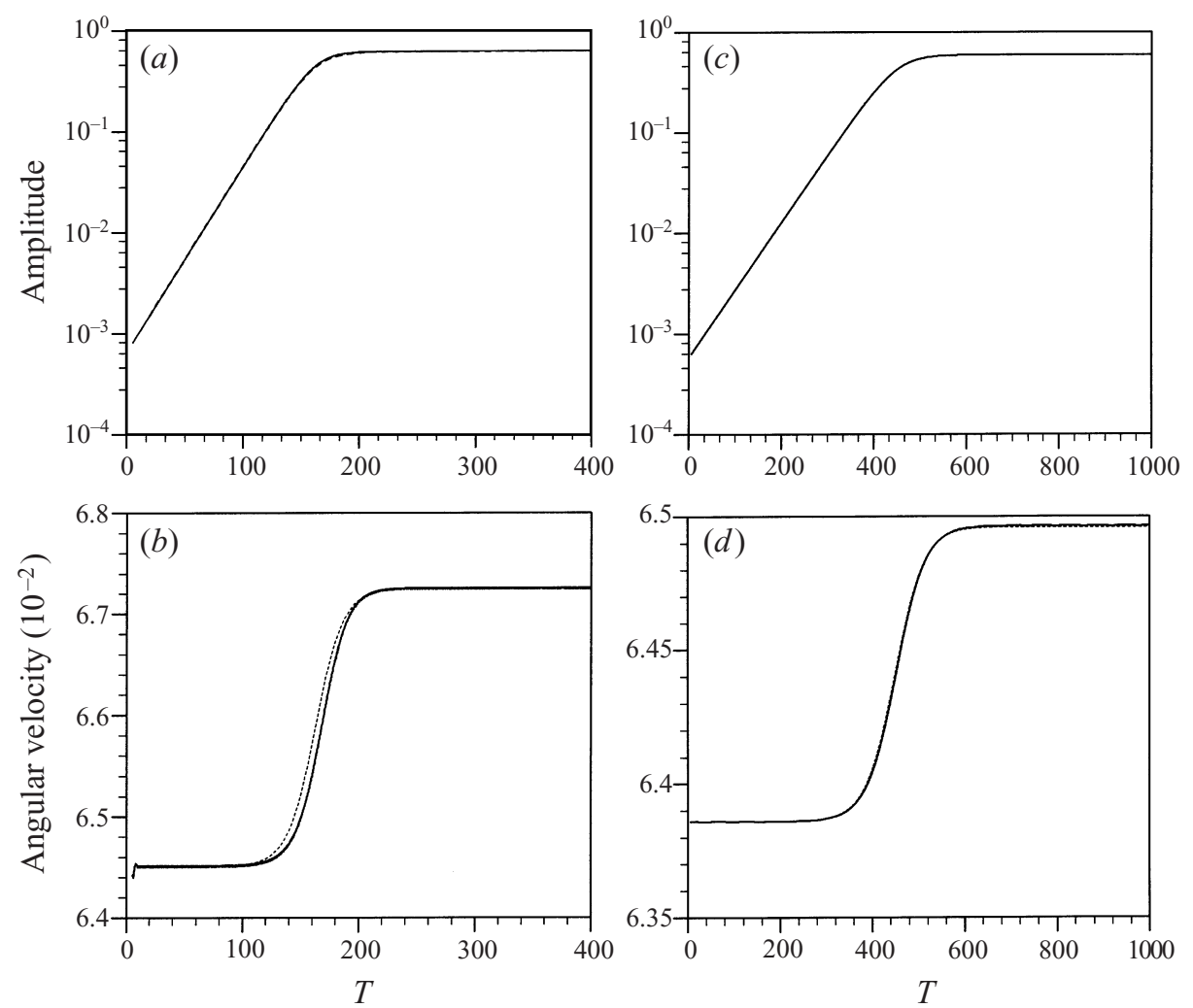

FIGURE 8. Evolution of amplitude and angular velocity of a mode 5 perturbation for $R e=89.32$, $a=3, \Gamma=6.5$, and cubic profile for $\Omega^{*}$. $(a, b)$ Free-slip boundary conditions; $(c, d)$ no-slip boundary conditions. Solid lines show results from full numerical simulation; dashed lines show fit to Landau equation.

In figure 8 , the evolution of the amplitude, $\hat{\omega}$, and the angular velocity, $\mathrm{d} \phi / \mathrm{d} t$, are shown as solid lines for $R e=89.32$ with both free-slip and no-slip boundary conditions. Based on the asymptotic behaviour of these two quantities, the values of the four real constants $\alpha_{r}, \alpha_{i}, \tilde{\xi}_{r}$, and $\tilde{\xi}_{i}$ can be determined. The results are summarized in table 1 . We note the good agreement between the values of $\alpha_{r}$ obtained from the simulations and the results from the linear analysis. In either case those values agree to $O\left(\left|v_{c}-v\right|\right)$, which equals 0.0003 and 0.001 for the no-slip and free-slip cases, respectively. With the constant coefficients determined, we are able to solve (5.2) and (5.3) for the theoretical predictions of the full dynamical evolution of $\hat{\omega}$ and $\mathrm{d} \phi / \mathrm{d} t$ (Drazin \& Reid 1991). The results of such calculations are shown in figure 8 as dashed lines. We note an excellent agreement in the no-slip case (the dashed lines are almost completely hidden), while the free-slip case shows a minor mismatch for the angular velocity. Equation (5.3) predicts an instantaneous coupling between the amplitude of the perturbation and the angular velocity, while figure $8(b)$ indicates a slight time lag between these quantities in the full simulation. It is not surprising that the agreement between the results from the asymptotic analysis and the full simulation is slightly worse in the free-slip case than in the no-slip case, since the value of the Reynolds number is further away from $R e_{c}$ in this case (see figure 6). 

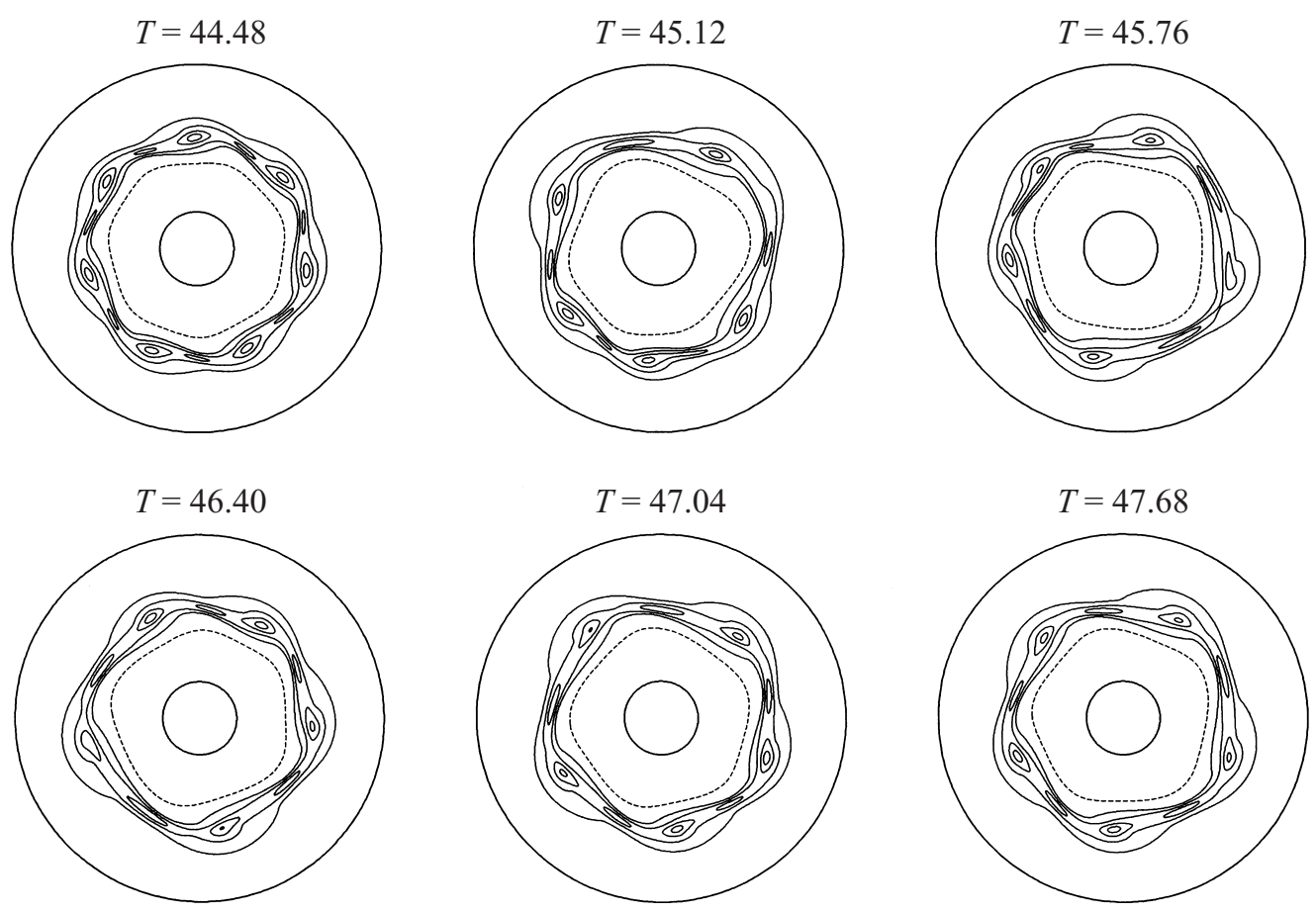

FIGURE 9. Symmetry-breaking transition during spin-up for $a=1.5, \Gamma=10, \operatorname{Re}(t=4)=84$, $\mathrm{d} R e / \mathrm{d} t=1.27$, cubic interpolation for $\Omega^{*}$, free-slip boundary conditions, and spectral resolution $M=N=256$. The figure shows the evolution of the vorticity field, $\omega$, where the solid contours indicate positive values of $\omega$ and dashed lines negative values. The difference between the contour levels is 50 , and the first contours levels are at \pm 25 thereby suppressing the zero contour.

\subsection{Spin-up and spin-down}

When $R e$ is increased further above $R e_{c}$ than in the previous subsection, a series of symmetry-breaking bifurcations occur. Each one of these is characterized by a reduction of the number of vortices in the system. In this subsection we will study the transition processes during spin-up and spin-down in some detail. Since we are not interested in investigating boundary layer processes per se in this section, freeslip boundary conditions are employed in all the runs. The runs were initiated at $R e$ slightly above $R e_{c}$, and $R e$ was kept constant for a period of time allowing the unstable mode of the system to saturate. After this start period, the Reynolds number was gradually increased at a constant rate. If a spin-down simulation was also being performed, the spin-up phase was followed by period of constant Reynolds number. During this period the system was allowed to settle into a well-defined state in which the transients caused by the spin-up phase have died out. This high-Re state may either be stationary or time-dependent, as described below. The spin-down phase follows the high-Re state, and the decreasing of the Reynolds number occurs at exactly the opposite rate as during spin-up.

Figure 9 shows a symmetry-breaking transition during spin-up in a case where $a=1.5, \Gamma=10, \operatorname{Re}(0 \leqslant t \leqslant 4)=84, \mathrm{~d} R e / \mathrm{d} t=1.27$, a cubic interpolation was used for $\Omega^{*}$, and the spectral resolution was $M=N=256$. During the transition process, at $t \approx 45$, two of the vortices are weakened so much that they lose their identities, and the first unstable mode of the system with seven equally distributed vortices is transformed into a state with five equally distributed vortices. From the time traces 


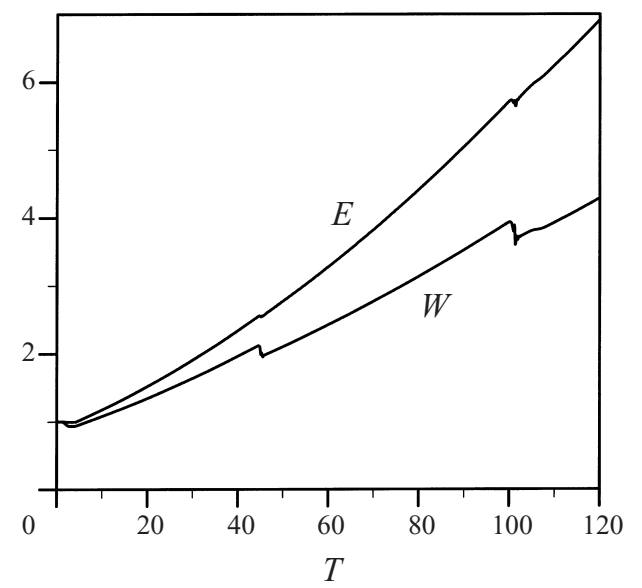

FIGURE 10. Temporal evolution of the total energy, $E$, and total enstrophy, $W$, for the run shown in figure 9.

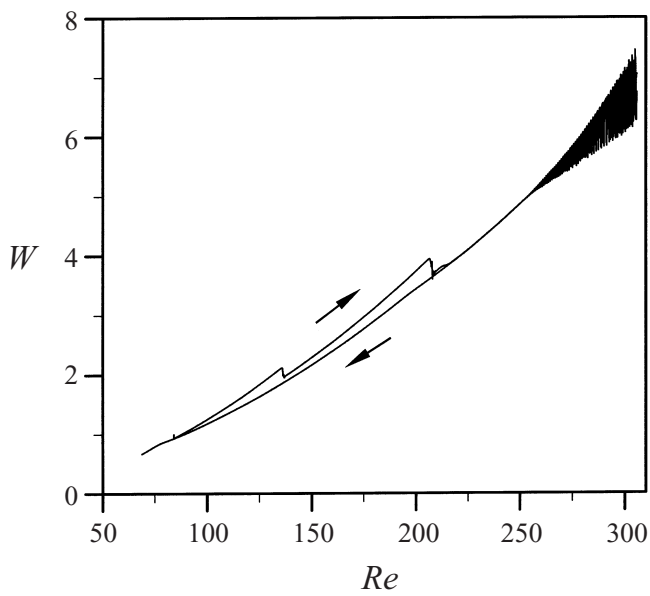

FiguRE 11. Hysteresis in the temporal evolution of the total enstrophy, $W$, during a complete spin-up and spin-down simulation for parameters similar to those in figures 9 and 10 .

of total energy, $E$, and total enstrophy, $W$, in figure 10 we see that this transition around $t=45$ is accompanied by clear drops in both $E$ and $W$. Since most of the energy of the system is stored in the non-rotational background flow, the relative drop in energy is lower than for the enstrophy. The other drops in $E$ and $W$ slightly before $t=100$ are caused by a $5-4$ transition.

In figure 11 we show a full spin-up/spin-down simulation for the same case as in figures 9 and 10 . Here we show the change of $W$ as a function of Re. Above $R e \approx 250$, a temporal state with both mode 4 and mode 2 is created. This state is further described in the next subsection. During spin-down the system shows a strong hysteresis staying in the mode 4 state with a gradually diminishing amplitude all the way down to $R e \approx 78$ where the axisymmetric state is restored.

This behaviour during spin-up and spin-down shares many features with the experiments of Rabaud \& Couder (1983) and Chomaz et al. (1988). In the laboratory experiments, transitions to states with fewer vortices during spin-up were also found 

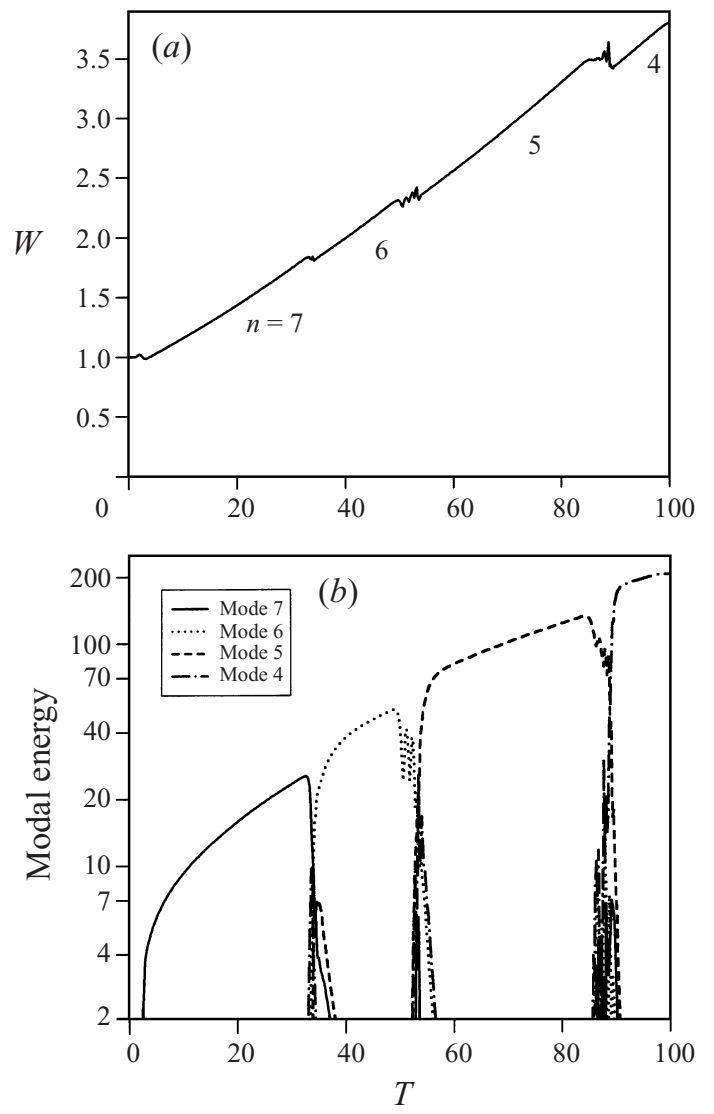

FIGURE 12. Spin-up with noise added to the forcing function. The noise amplitude $\varepsilon=0.1$ while the other parameters are the same as in figures 9-11. (a) Temporal evolution of the total enstrophy, $W$; (b) temporal evolution of the energy content in the Fourier modes 4-7.

and a strong hysteresis effect occurred during spin-down. However, in the experiments the spin-up transitions at low spin-up rates reduced the number of vortices by one, while our 7-5 transition shows a change in number by 2 . Furthermore, during spindown the laboratory experiments demonstrated transitions during which the number of vortices was increased by one. These transitions continued until the same number of vortices was reached as predicted by the first bifurcation in the asymptotic analysis. The range of $R e$-values over which these transitions occurred during spin-down in the experiments was very narrow and close to $R e_{c}$. The numerical simulations by Chomaz et al. (1988) also demonstrated an increase in the number of vortices during spindown. It is not clear whether their numerical simulations were performed at constant spin-down rate (reducing $R e$ by a small and constant amount each time step) or if $R e$ was decreased in larger steps followed by longer periods of constant $R e$. In any case, the spin-down simulation shown in figure 10 of Chomaz et al. (1988) demonstrates a transition from 3 to 5 vortices. This transition occured via an axisymmetric state, which is rather surprising and in contrast to the experiments where the transition occured via splitting of elongated vortices.

To make the numerical simulations behave more like the laboratory experiments, noise was added to the forcing function. In the first attempt, this noise was applied 


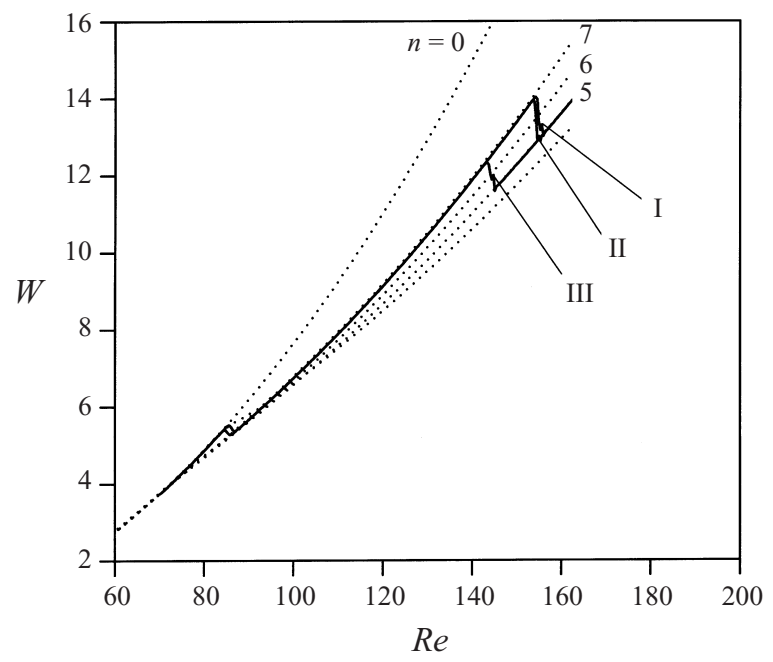

FIGURE 13. Effect of resolution and noise on a mode 7-5 transition during spin-up. The curves show the variation of the total enstrophy, $W$, as the Reynolds number, $R e$, is increased. A tanh-profile was used for $\Omega^{*}(r), a=1.5, \Gamma=12$, and $\mathrm{d} R e / \mathrm{d} t=2.12$. The Roman numerals on the three solid curves indicate: I resolution $M=N=256$, noise amplitude $\varepsilon=0 ;$ II $M=N=128, \varepsilon=0$; and III $M=N=128, \varepsilon=10^{-3}$. The dotted lines show the relation between $W$ and $R e$ when the symmetry of the flow is artificially kept by only allowing the evolution of the Fourier mode with a given $n$, indicated on the figure, and its higher harmonics.

in a region of width $h$ centred around $r=a$, and was produced by multiplying the forcing field, $\omega^{*}(r)$, with a factor $(1+\varepsilon \Delta(t))$, where $\Delta(t)$ is a random number with unit amplitude re-chosen at each time step. Figure 12 shows the result from a spin-up simulation for a case similar to that in figures 9-11 and with $\varepsilon=0.1$. In figure 12(a) clear drops in $W$ are seen at each transition, and in figure $12(b)$ it is seen that a full sequence of distinct Fourier modes 7-6-5-4 is obtained. A spin-up simulation was also performed with a lower noise amplitude $\varepsilon=0.05$, but in this case mode 7 transformed directly into a mode 5 , just as without noise. During spin-down mode 4 still persists all the way down until the axisymmetric mode is restored, even for a high noise amplitude $\varepsilon=0.1$. We will return to the spin-down and the missing transitions to higher mode numbers after investigating the spin-up transitions in more detail.

\subsubsection{Spin-up}

In figure 13 we show the effect of noise and of spatial resolution on the global state of the flow. The figure shows the change in $W$ as $R e$ is increased and in all cases shown a tanh-profile was used in the angular velocity forcing function, $a=1.5$, $\Gamma=12$, and $\mathrm{d} R e / \mathrm{d} t=2.12$. The Roman numerals on the three solid curves indicate: I resolution $M=N=256$, noise amplitude $\varepsilon=0$; II $M=N=128, \varepsilon=0$; and III $M=N=128, \varepsilon=10^{-3}$. The dotted lines show the relation between $W$ and $R e$ when the symmetry of the flow is artificially kept by only allowing the evolution of the Fourier mode with a given $n$, indicated on the figure, and its higher harmonics. By comparing the solid curves marked by Roman numerals, we note that even the small level of noise used in III causes a large change in the value of $R e$ at transition. On the other hand, increasing the resolution from $M=N=128$ to $M=N=256$ has very little effect on the value of $R e$ at transition, indicating that the resolution is adequate. Although the dynamics of the flow is very delicate near a transition, figure 13 also 


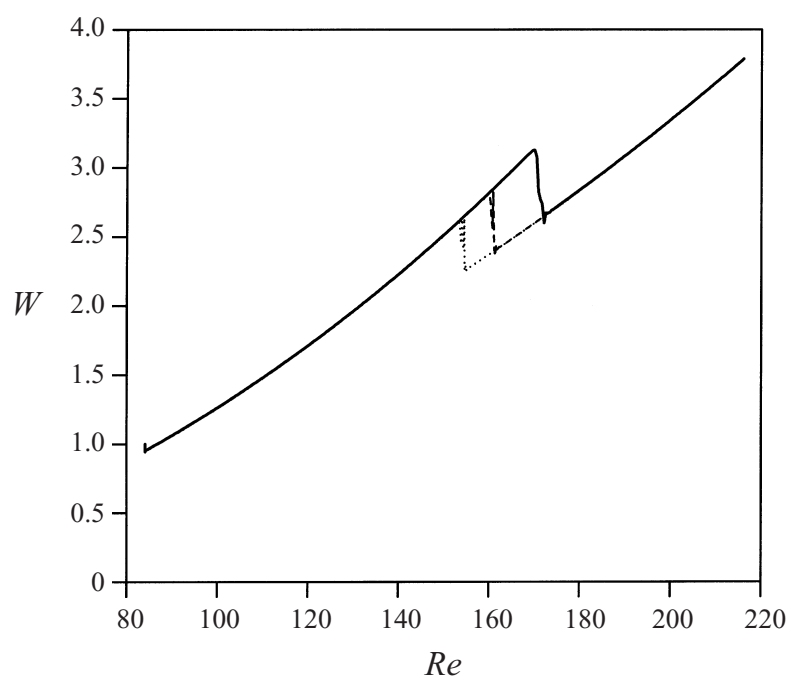

FIGURE 14. Effect of spin-up rate on the value of Re at a mode 6-3 transition. A tanh-profile was used for $\Omega^{*}(r), a=1.5, \Gamma=10, M=N=128$, and $\varepsilon=0$. The solid curve shows the result for $\mathrm{d} R e / \mathrm{d} t=2.55$, the dashed line for $\mathrm{d} R e / \mathrm{d} t=1.27$, and the dotted line for $\mathrm{d} R e / \mathrm{d} t=0.64$.

shows that the flow is attracted to a state with well-defined $W(R e, n)$ between the transitions. The value of $W$ for a given $R e$ and $n$ seems to be the same, regardless of noise, even for the flows in which the azimuthal symmetry is kept artificially constant. Similar curves to those in figure 13 are also obtained for $E$ but with less pronounced splitting between states with different $n$.

The dependence of the value of $R e$ at transition on the spin-up rate is shown in figure 14. Here, we show results from flow simulations with a tanh angular velocity forcing profile, $a=1.5, \Gamma=10, M=N=128$, and $\varepsilon=0$. On all three curves, the drops in $W$ are connected to a mode 6-3 transition. Such transitions are also seen in the experiments by Rabaud \& Couder (1983) when the spin-up is not small (see their figures 13 and 14). The solid curve in figure 14 shows the result for $\mathrm{d} R e / \mathrm{d} t=2.55$, the dashed line for $\mathrm{d} R e / \mathrm{d} t=1.27$, and the dotted line for $\mathrm{d} R e / \mathrm{d} t=0.64$. We notice that lowering the spin-up rate makes the transition occur at an earlier stage with lower $R e$. As in figure 13, we also see that $W$ is a well-defined function of $R e$ once the symmetry of the flow is determined. A similar behaviour is found for $E$.

The results from the different spin-up simulations presented in this section show that the adjustment of the flow to changes in $R e$ occurs on two very different time scales depending on whether the flow has a fixed symmetry or not. As long as the symmetry is fixed with a given azimuthal mode number, $n$, the adjustment of the flow to changes in $R e$ is very rapid (almost instantaneous), at least much faster than the time scales related to the spin-up rates used in our simulations. This gives rise to unique relations between the global flow quantities (such as $E$ and $W$ ) and $R e$ even in cases when $R e$ is changing rapidly in time. The values of the global quantities in these states with fixed symmetry are not sensitive to noise in the forcing function.

On the other hand, if the flow has become sufficiently unstable (for reasons not well understood) at a given mode number, $n$, a symmetry-breaking transition sets in, and this transition process occurs on a much slower time scale. During spin-up, the transition process leads to a reduction in the number of vortices in the flow, and we have shown that it also leads to clear drops in global energy, $E$, and global enstrophy, 
$W$. By monitoring video animations of the numerical results for the transition process with high time resolution, we found that the reduction in the number of vortices is not caused by normal vortex pairing. Instead, some of the vortices weaken, in the sense that their amplitudes diminish, and, eventually, they lose their identities. The remaining vortices move in the azimuthal direction into the vacant spaces from both sides and the transition process is completed when the remaining vortices have equal sizes and are evenly distributed around the annulus.

The onset of the instability that causes the symmetry-breaking transitions is not easily determined. Adding noise to the forcing function lowers the value of $R e$ at which the transition occurs in a spin-up simulation. By adding a fairly high level of noise, with amplitude $\varepsilon=0.1$, we were able to produce a sequence of transitions in which the number of vortices was reduced by one each time. Without noise, however, the transitions occurred at higher values of $R e$, and when they finally did occur, they often caused more than one vortex to vanish. Thus, a subharmonic bifurcation from 6 to 3 vortices can be observed, like the one described in connection with the results in figure 14. The spin-up rate has a clear effect on the value of $R e$ at which a transition occurs, with lower spin-up rates resulting in lower values of $R e$ at transition.

\subsubsection{Spin-down}

We now return to the spin-down simulations described above. Based on the experience obtained from the spin-up simulations described above, we expect that 'reverse' transitions to higher mode numbers during spin-down are only possible when $E$ and $W$ can drop to lower levels. This, in turn, requires that the dotted lines in figure 13 indicating $W$ for flows with fixed $n$ (and the similar curves for $E$ ) cross each other for low values of $R e$. This actually happens, as shown in figure 15 . Here, the different broken lines indicate the variation with $R e$ of $E_{n}$, for different mode numbers $n$. In order to make the crossings of these lines visible, we have plotted $\left(E_{n}-E_{0}\right) / E_{0}$, and since $E_{0}$ is larger in magnitude than the other $E_{n}$ the result is negative. Now, although a mode transition can be energetically favourable it is not certain that it will occur. Indeed, we were not able to see any reverse transitions in the simulations shown in figure 11 nor in the corresponding simulations with noise, even with noise amplitudes as high as $\varepsilon=0.1$. We also tried to stop the spin-down and keep Re constant at different values between 90 and 80 with and without noise, but the mode $n=4$ persisted.

The noise perturbation used so far has consisted of a time-dependent constant multiplied on the force function which has a radial dependence only. A second noise function was now employed introducing an azimuthal perturbation. The total forcing takes the form $\omega^{*}(r)(1+\varepsilon \xi(\theta))$, where

$$
\xi(\theta)=\sum_{n=1}^{10} \mathrm{e}^{\mathrm{i} n \theta} \mathrm{e}^{\Theta_{n}(t)},
$$

and $\Theta_{n}(t)$ is a random phase re-chosen for each $n$ at each time step.

In figure 15 the solid line shows the result of a spin-down simultation with parameters similar to those in figures 9-12 and with $\mathrm{d} R e / \mathrm{d} t=-0.758$ and $\varepsilon=0.05$. The small oscillations in the solid line are caused by the external noise. Now, we see both a mode 4-5 transition and a 5-7 transition. These transitions occur at slightly lower $R e$-values than allowed based on pure energy minimization, but on the other hand at $R e$-values that are distinctly higher than the values determined by marginal 


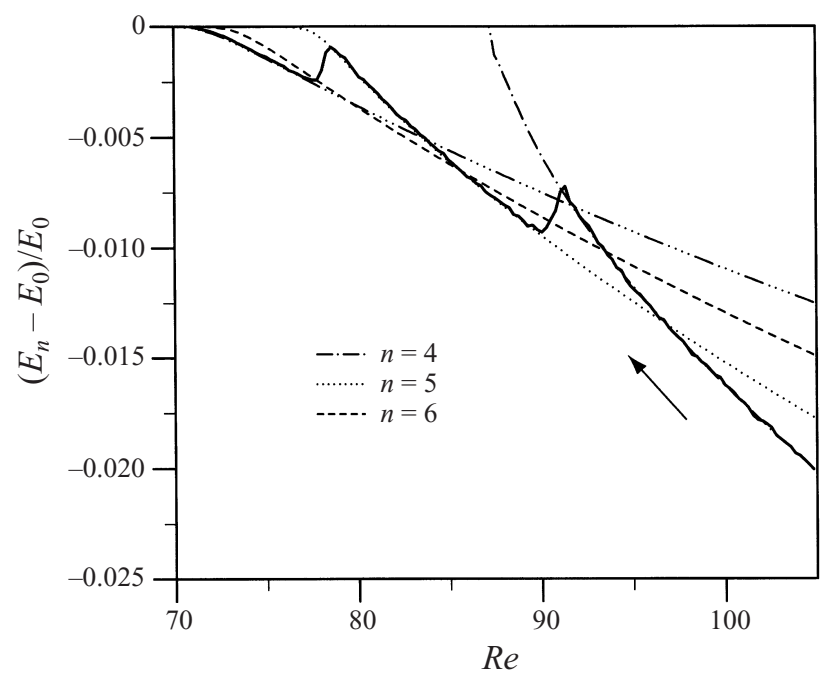

FIGURE 15. Spin-down transitions for parameters similar to those in figures 9-12. The broken lines show the normalized energy, $\left(E_{n}-E_{0}\right) / E_{0}$, of flows with artificially constant mode number, $n$, with the line coding shown in the figure legend. The solid curve shows the normalized energy of a full simulation with a noise function including azimuthal perturbations. The arrow shows the direction of change in $R e$.

stability, found approximately by the intersection of broken lines with the horizontal axis.

Figure 16 shows contour plots of $\omega(r, \theta, t)$ during the $4-5$ transition in figure 15 . We see that the transition occurs without passing through a transient axisymmetric state. This is in agreement with the experimental results but different from the numerical result of Chomaz et al. (1988) which seems to be an artifact caused by their rather crude numerical method.

\subsection{Large values of $R e$}

As we have seen in the previous subsections, increasing the Reynolds number leads to bifurcations from the axisymmetric state to states of rigidly rotating waves of progressively lower order of symmetry. At sufficiently large values of $R e$, temporal states with several azimuthal modes simultaneously present can be formed. In figures 17 and 18 we show vorticity contour plots of two such temporal states during one period of oscillation, and in figures 19 and 20 we show the corresponding temporal evolution of the enstrophy, $W$. These temporally periodic states are obtained at constant $R e$ following a spin-up phase with $\mathrm{d} R e / \mathrm{d} t=2.54$ and after the transients have died away, as seen in figures 19 and 20. The states are characterized as modulated waves with $p$ vorticity peaks (with $p=4,3$, respectively) and order of symmetry $s<p$ (with $s=2,1$, respectively). Unlike the rotating waves which can be made stationary by a change to an appropriate rotating frame, these states are oscillatory in a frame co-rotating with the mean angular velocity of the peaks. Moreover, if the minimum period of that oscillation is $T$, then the modulated waves can be mapped onto themselves by a rotation by angle $2 \pi l / p$ after time $l T / p$ (with $l=0,1$, respectively). We also note that the oscillatory pattern in figure 17 is a result of a bifurcation from a non-oscillatory state of symmetry $p=4, s=2$ during spin-up.

Following the discussion in Rand (1981), we say that figure 17 shows a modulated wave of symmetry type $p=4, s=2, l=0$ while figure 18 shows a modulated wave 

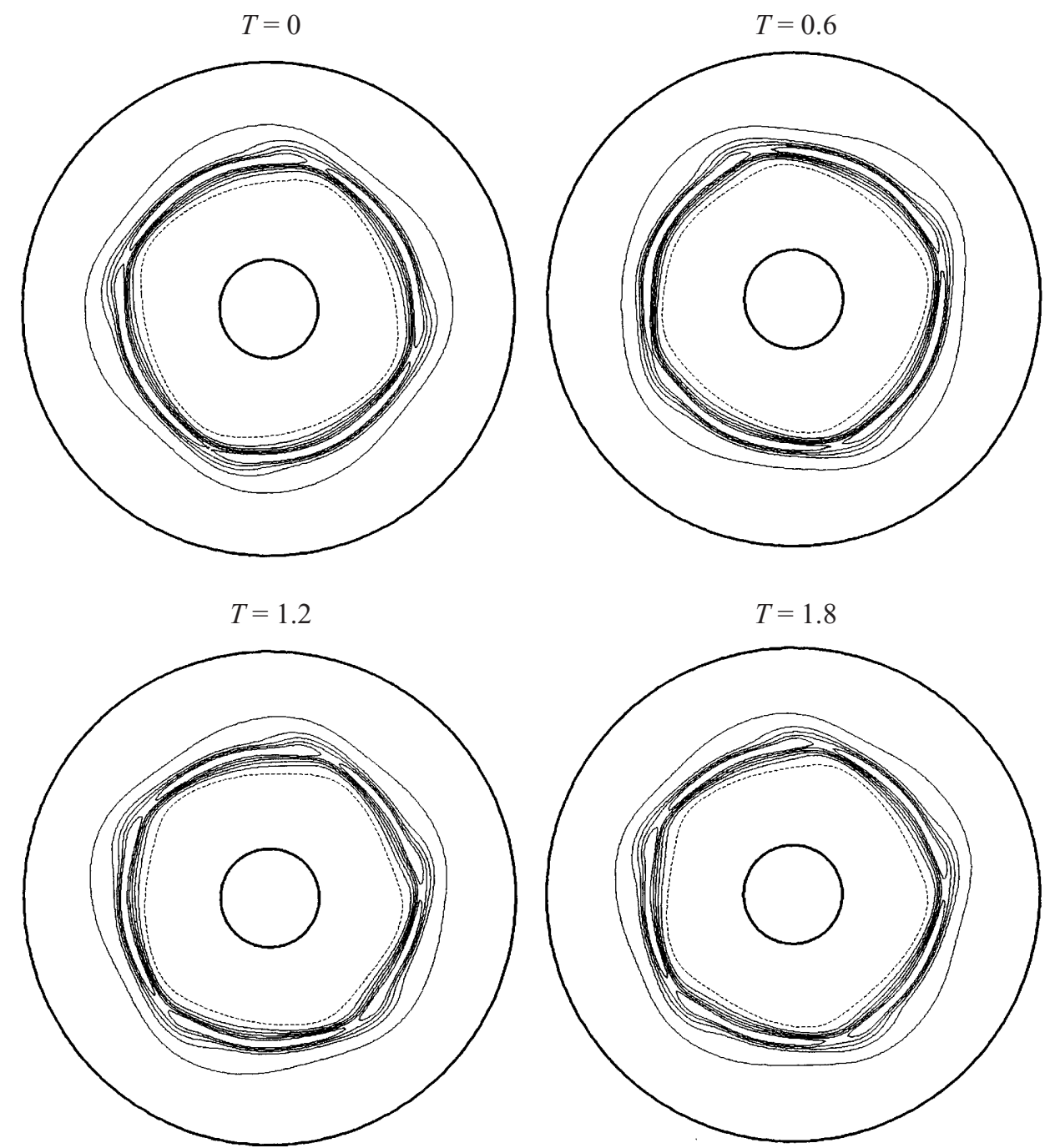

FIGURE 16. A mode 4-5 transition during spin-down for parameters similar to those in figure 15. The figure shows the evolution of the vorticity field, $\omega$, where the solid contours indicate positive values of $\omega$ and dotted lines negative values. The difference between the contour levels is 30 and the first contours are at \pm 15 thereby suppressing the zero contour.

of symmetry type $p=3, s=1, l=1$. According to Rand's (1981) classification, the first case cannot be the result of a super- (or sub-) critical Hopf bifurcation, while the second does fit the requirements for such a bifurcation to be the originating mechanism. It seems likely that the situation depicted in figure 17 arises due to the simultaneous creation of two counter-rotating waves, due to two eigenpairs crossing the imaginary axis. We have not yet seen vacillating waves with two clear frequencies in the co-rotating frame, as predicted by Rand, except at very high values of $R e$ when single vortex states with strong boundary influence form (see below). The rich dynamical behaviour associated with the transitions to modulated (and presumably to vacillating) waves will be the subject of a subsequent high-resolution investigation. We would like, however, to point out that the modulated waves described here do 

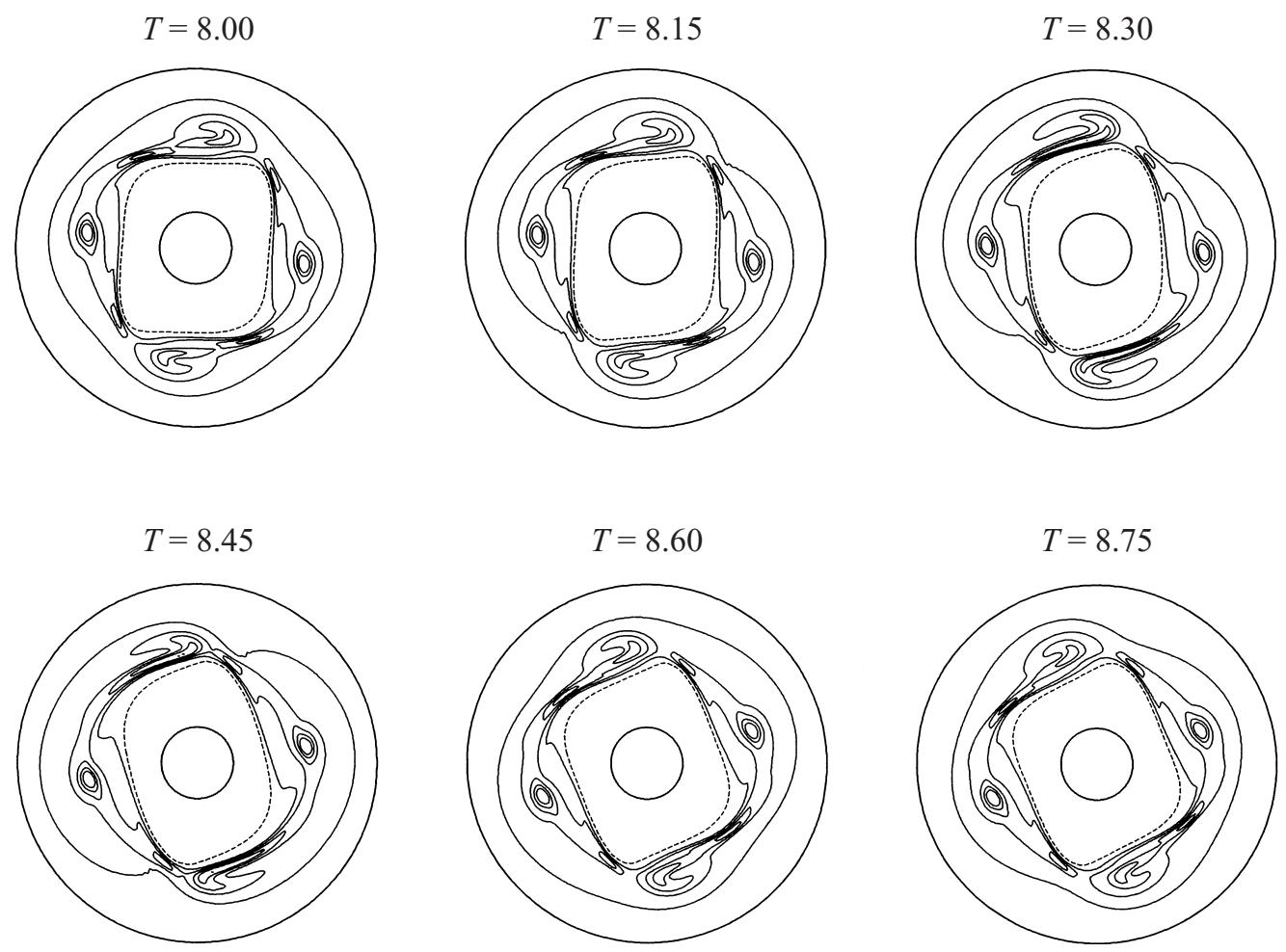

FIGURE 17. Vorticity field for a full period of a temporal mode with $(p, s, l)=(4,2,0)$. The simulation parameters are: $a=1.5, \Gamma=10, \operatorname{Re}=277.28, M=N=256$, cubic interpolation for $\Omega^{*}$ and free-slip boundary conditions. The difference between contour levels is 60 , with the first levels at \pm 30 thereby suppressing the zero contour.

fit some of the requirements that suggest the possibility of chaotic fluid mixing near their separatrices. In particular, the $p=3$ modulated wave seems to fit some of the properties of the oscillating vortex flows discussed by Wiggins (1992) as agents of chaotic fluid transport. A detailed study of the Melnikov structure of weakly modulated waves is currently underway and we will report on it elsewhere.

We have also performed simulations for values of Re between 1000 and 2000, and in this regime very complicated states are formed. The evolution of the flow at these high Reynolds numbers is strongly affected by the location of the walls and by the boundary conditions. Figures 21 and 22 show flows at $R e=1528$ with free-slip and no-slip boundary conditions, respectively.

In the free-slip case, an unsteady single vortex state is formed. This state is modulated with an azimuthal mode $n=2$ and energy is constantly transferred from one distinct vortex to another. At the same Reynolds number, the no-slip case shows a steady, single vortex state which continuously generates eruptions of vorticity from the inner wall. The turbulent flow generated by these eruptions is localized within a turbulent 'bubble' located on the opposite side of the inner cylinder to the steady vortex.

It is clear that, when modelling real experiments at high values of $R e$, it should be considered whether the model equations (2.2) and (2.3) should be extended to include new physical effects (such as flow out of the plane). The proper boundary conditions are also crucial since they have strong influence on the flow at high $R e$, 

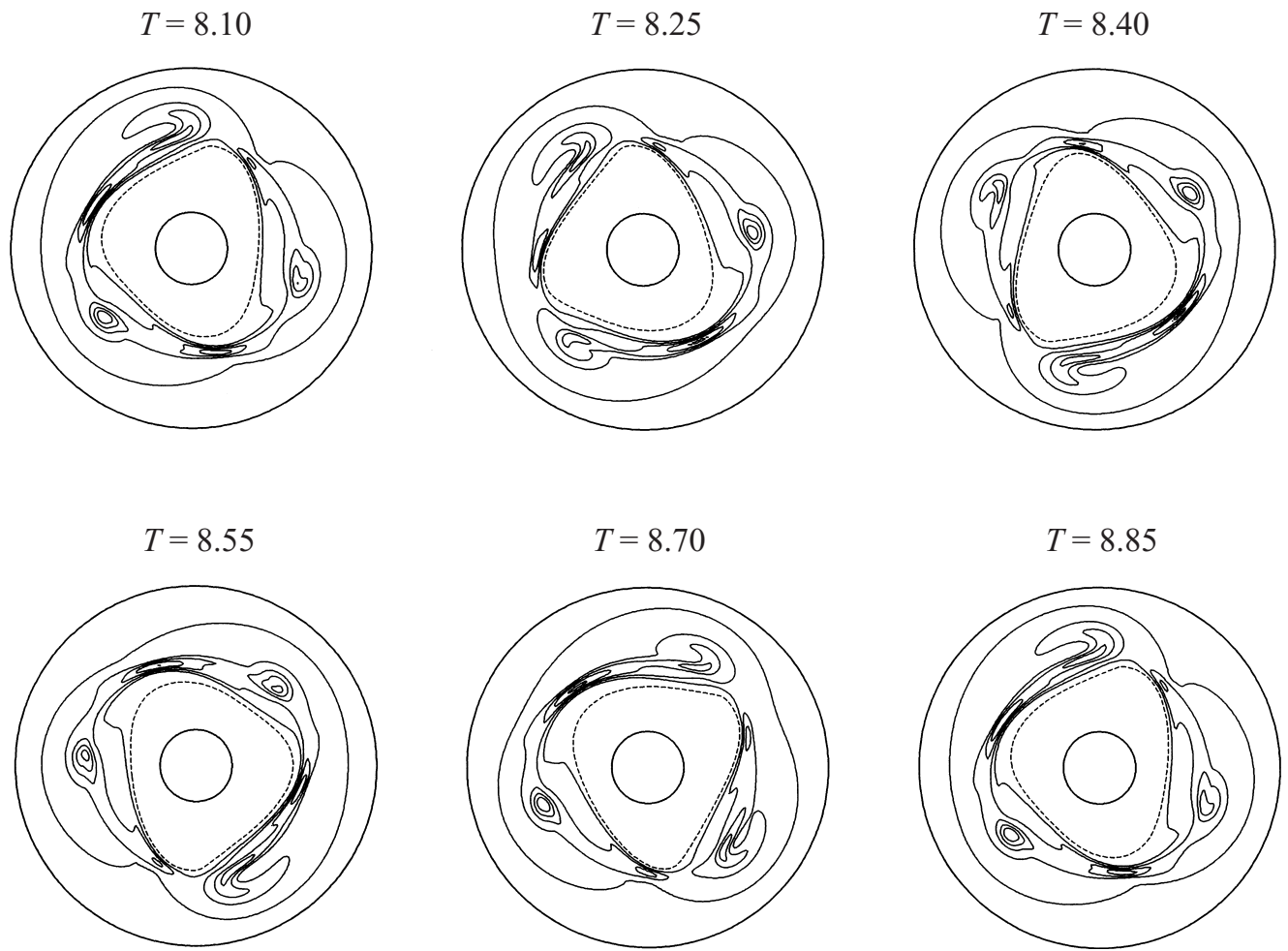

Figure 18. Same as figure 17 , but for a temporal mode with $(p, s, l)=(3,1,1)$ obtained at $R e=282.37$.

as demonstrated in the last two simulations. The correct modelling requires careful analysis of the specific experiment under investigation.

\section{Conclusion}

In this paper, we have studied in detail a number of different dynamical properties of forced shear layers in an annular geometry. As mentioned in the introduction, annular shear layer flows are found in a large variety of physical systems. Despite the differences in flow media (gas, fluid, quasi-neutral and non-neutral plasma, etc.) and the corresponding forcing mechanisms producing the shear layer, the qualitative behaviour of the flows is remarkably similar. Thus, the flows are axisymmetric for low values of $R e$, but become unstable at a critical $R e=R e_{c}$ to perturbations with a well-defined azimuthal mode number, $n_{c}$. For $R e$ values slightly above $R e_{c}$, the unstable pertubation evolves into a braid of $n_{c}$ vortices along the circular shear layer. With increasing $R e$, these vortices transform into new arrangements with decreasing order of symmetry, and with subsequent decreasing of $R e$ the flows demonstrate strong hysteresis.

We have chosen to work with a dynamical equation that was derived by Chomaz et al. (1988) for a particular flow experiment. This choice allowed us to cross-validate the results from our asymptotic analysis and numerical simulations by comparison with well-documented experimental results (Rabaud \& Couder 1983; Chomaz et al. 1988). In $\S 2$, we described the model equations and presented the precise form of the forcing profiles used in our paper. We also described the inclusion of both no- 


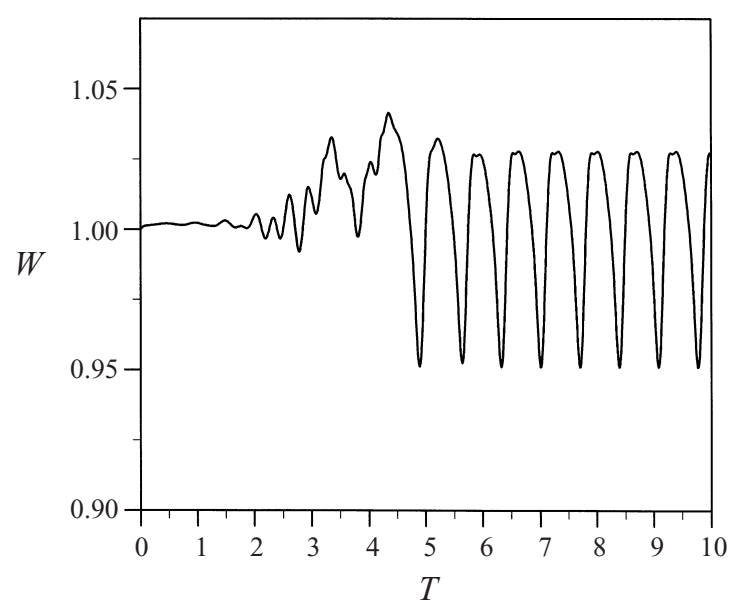

FIGURE 19. The evolution of global enstrophy, $W$, in a temporal state with both azimuthal modes $n=4$ and $n=2$ present. The evolution of vorticity field in the oscillatory phase is shown in figure 17.

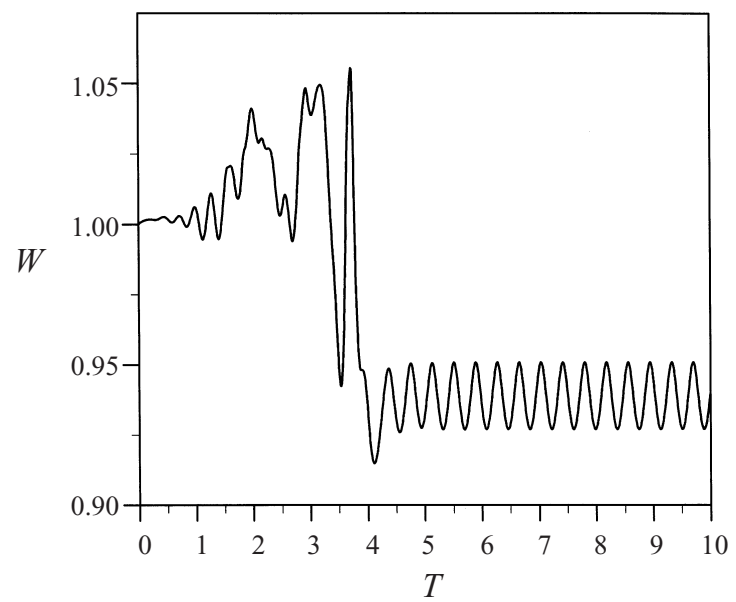

FIGURE 20. The evolution of global enstrophy, $W$, in a temporal state with both azimuthal modes $n=3$ and $n=1$ present. The evolution of vorticity field in the oscillatory phase is shown in figure 18.

slip boundary conditions and different free-slip models in the Poisson and Helmholtz equations through direct use of a spectral expansion of the flow fields in a FourierChebyshev series. The no-slip boundary conditions were used in the comparisons with the fluid experiments, while the free-slip models were introduced to generically model annular shear flows without wall-induced vorticity, such as plasma flows. In $\S 2$, we also gave expressions for the temporal evolution of energy, enstrophy, and angular momentum of the total flow to be used as accuracy checks in the numerical simulations.

The asymptotic analysis described in $\S 3$ was carried out in a combined analytical and numerical approach. The spectrum of the linear problem was calculated numerically, and the dominant eigenvalues were shown to be insensitive to perturbations. The linear analysis gave values for $R e_{c}$ and $n_{c}$ that are in good agreement with the experimental values. The perturbation expansion was carried out to third order, at 

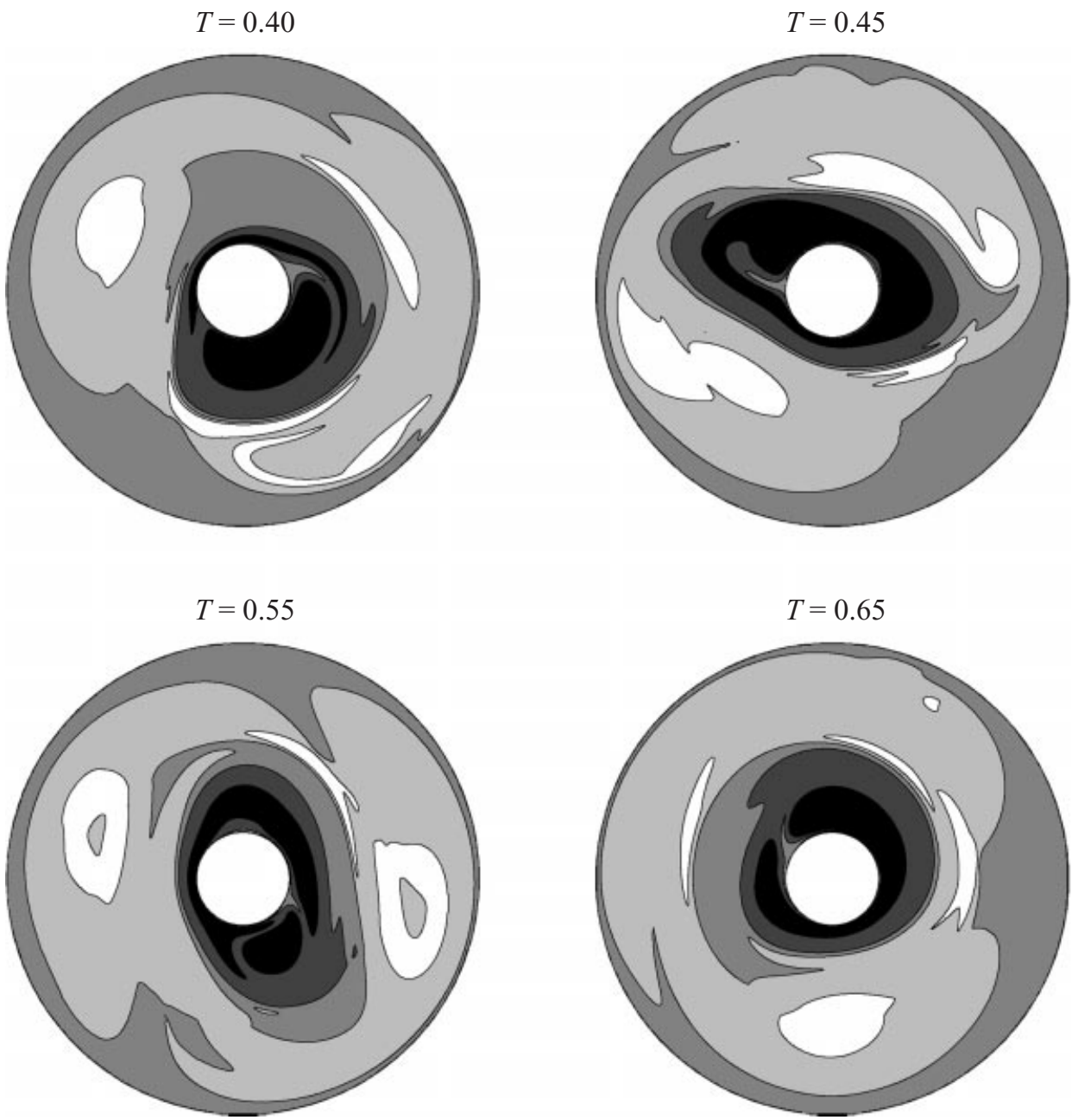

FIGURE 21. The evolution of the vorticity field at $R e=1528$ with free-slip boundary conditions. Dark regions represent low levels of vorticity and white regions high levels. A cubic profile was used in the angular velocity forcing, $a=1.5, \Gamma=10$, and $\varepsilon=0$.

which resonant terms appear. Application of the Fredholm alternative theorem lead to a Landau equation describing the saturation behaviour of the neutral mode. Unfortunately, the variational problems occurring at the second- and third-order levels are numerically ill-conditioned so direct numerical solutions do not give accurate results. As mentioned in $\S 3$, we are at present testing different conditioning methods to make these problems solvable with high accuracy.

The spectral method used in our numerical simulations was briefly described in $\S 4$ and results from the simulations presented in $\S 5$. Our first simulations were focused on the near linear problem. Here, we demonstrated a clear supercritical bifurcation behaviour for flows subjected to both no-slip and free-slip boundary conditions, and very close agreement was obtained when comparing the results from the full simulations with those from the linear analysis. When comparing with the results from fluid experiments, we obtained a much better agreement than previously obtained by others, and we ascribe this close agreement to the high accuracy of 

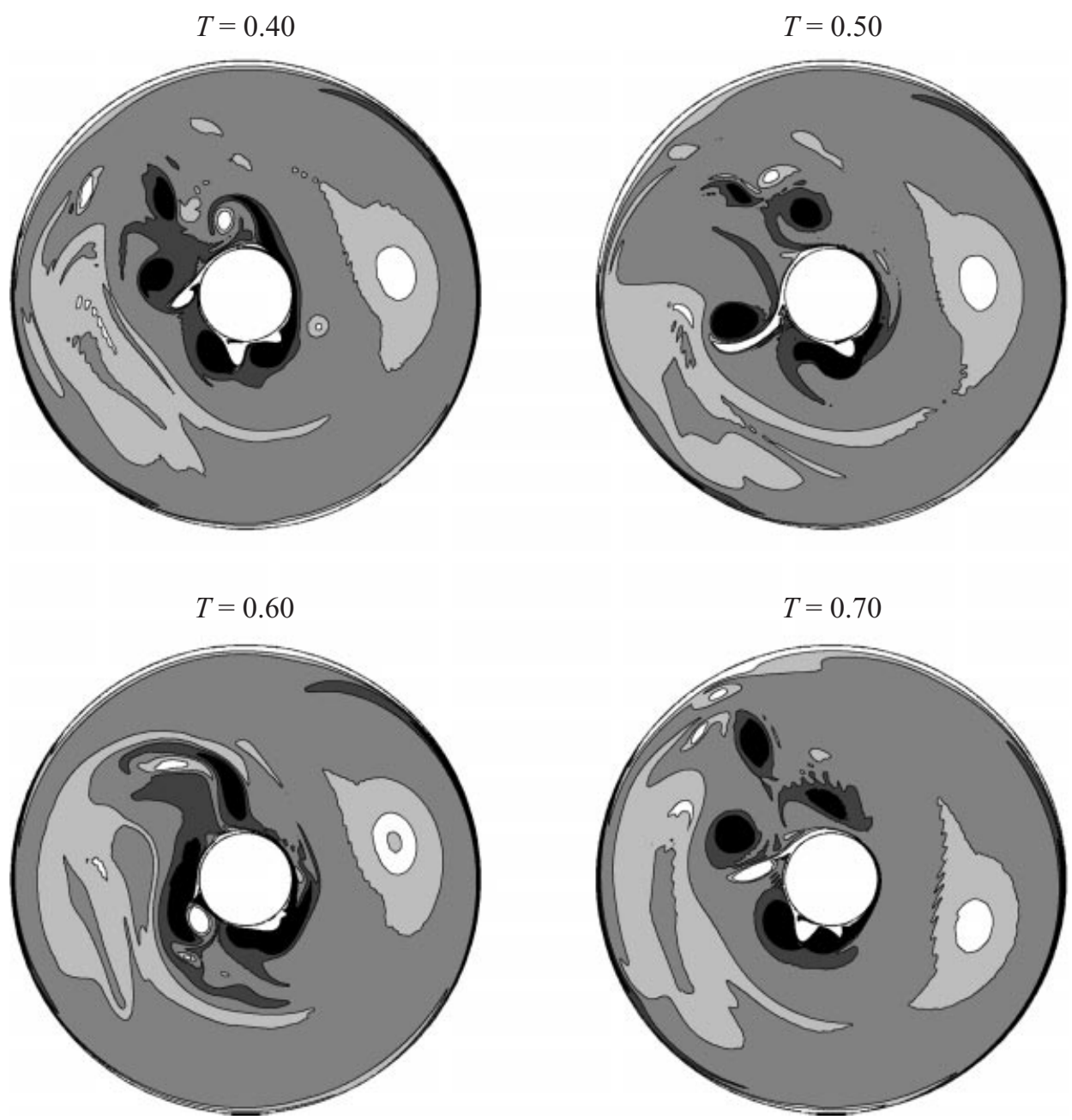

FIGURE 22. Same as figure 21, but with no-slip boundary conditions.

our numerical solution. While the quantitative agreement between the experimental results and the results from previous numerical studies by others were so rough that the validity of the model equations could not be definitely confirmed, the close agreement we have presented in this paper demonstrates that the model equations are fully adequate for describing the experimentally observed flow dynamics, at least for low to moderate $R e$-values. In particular, our results show that three-dimensional effects are not important for these fluid flows.

The temporal evolution of both the total energy and the total enstrophy in the system during a transition away from the axisymmetric state was monitored, and clear drops in both quantities were observed. This indicates why the new state is preferred. We are able to monitor the temporal evolution of the content in each azimuthal mode of both energy and enstrophy, and time traces of these modal quantities have given us accurate information on how the various modes either grow and saturate, or die away. By following the growth and saturation of a perturbation corresponding to the most unstable mode, we were able to determine the complex Landau coefficients, and using 
these coefficients we demonstrated that the Landau equation models the temporal behaviour of the mode saturation obtained by the full simulation very closely.

Numerical studies of the behaviour of the flow during spin-up and spin-down were presented in $\S 5.2$. In an attempt to give a quantitative description of the flow evolution both during transitions and in between, we again found it very useful to follow the evolution of the total enstrophy, or energy, as well as the distribution of the enstrophy, or energy, on the different azimuthal modes. Although the precise dynamical evolution of the flow, including the details of the symmetry-breaking bifurcations, depends on a number of different parameters, we were able to extract some generic properties which characterize the system even far away from equilibrium during both spin-up and spin-down. From time traces of the total enstrophy, or energy, we observed that the adjustment of the flow to changes in $R e$ occurs on two very different time scales. Which of the two time scales is relevant depends on whether the flow evolves with unchanged symmetry or not.

As long as the symmetry (number of vortices) is constant, the adjustment of the flow to changes in $R e$ is very rapid (almost instantaneous), at least much faster than the time scales related to the spin-up and spin-down rates used in our simulations. This gives rise to unique relations, independent of history, between global quantities (such as $E$ and $W$ ) in a flow with a given number of vortices and $R e$, even in cases when $R e$ is changing rapidly in time. The values of the global quantities in these 'states of attraction' for given $n$ and $R e$ appear to be unique and insensitive to noise for a particular flow geometry, even for flows in which the azimuthal symmetry is kept artificially constant.

On the other hand, during transition from one symmetry type to another the flow evolves on a much longer time scale. During spin-up, the transition process leads to a reduction in the number of vortices, and we have shown that this is accompanied by clear drops in both $E$ and $W$. The onset of the instability that causes the symmetrybreaking transitions is not easily determined. Adding noise to the forcing function or increasing the spin-up rate both have the effect of lowering the value of $R e$ at which transition occurs. If a transition during spin-up is postponed for some reason, for example by spinning up very rapidly, the number of vortices in the flow may be reduced by more than one when a transition finally occurs.

A drop in $E$ and $W$ seems to be the driving mechanism behind all the mode transitions during both spin-up and spin-down. Although it is not possible to separate the drops in $E$ from those in $W$, as they occur simultaneously, we find it most natural if a minimization of energy is the main cause of the transitions. We have seen, however, that although a mode transition is possible from an energy minimization viewpoint, it may not occur. Especially during spin-down the flow seems very reluctant to make a mode transition and only by adding azimuthal perturbations to the noise function were we able to trigger a transition. We would like to emphasize that when these distinctions between the effects of various types of external perturbations are possible it is due to the very high accuracy of our spectral method.

We also addressed the properties of two-dimensional shear flows at high Reynolds numbers, where temporal states are formed. Simulations revealed the existence of both regular temporal states, in which a few modes are simultaneously present, and states with chaotic temporal behaviour. Contrary to the flows at lower Re, these high Reynolds states are very sensitive, also qualitatively, to changes in boundary conditions. It can be expected that modelling real experiments at high values of Re will require extensions to the model equations used in this paper. The precise way in which the model equations should be modified will require a detailed physical 
description of the particular set-up, and we anticipate that the results from such simulations will be less general than the ones presented here.

During the course of work reported in this paper, two of the authors (K. B. and E. A. C.) visited Risø National Laboratory and another of the authors (J. P. L.) visited the University of New Mexico - the hospitality of both institutions during these visits is gratefully acknowledged. The authors are also grateful to J. S. Hesthaven for many valuable discussions on various aspects of spectral methods. This work was supported by DOE Grant DE-FG03-92ER25128 and by the Danish Research Councils.

\section{REFERENCES}

Antonova, R. A., Zhvaniya, B. P., Lominadze, D. G., Nanobashvili, D. I. \& Petviashvili, V. I. 1987 Use of a rapidly rotating shallow liquid to model vortices in a homogeneous magnetized plasma. Sov. J. Plasma Phys. 13, 765-768.

Batchelor, G. K. 1967 An Introduction to Fluid Dynamics. Cambridge.

Bergeron, K. 1993 The evolution of two-dimensional circular shear layers. PhD thesis, University of New Mexico, Albuquerque.

Chomaz, J. M., Rabaud, M., Basdevant, C. \& Couder, Y. 1988 Experimental and numerical investigation of a forced circular shear layer. J. Fluid Mech. 187, 115-140.

Churilov, S. M. \& Shukhman, I. G. 1992 Weakly nonlinear theory of the alternation of modes in a circular shear layer. J. Fluid Mech. 243, 155-169.

Coutsias, E. A., Bergeron, K., Lynov, J. P. \& Nielsen, A. H. 1994 Self organization in 2D circular shear layers. AIAA Paper 94-2407, 11 pp.

Coutsias, E. A., Hagstrom, T. \& Torres, D. 1995 An efficient spectral method for ordinary differential equations with rational function coefficients. Math. Comput. 65, 611-635.

Coutsias, E. A., Hagstrom, T., Hesthaven, J. S. \& Torres D. 1996 a Integration preconditioners for differential operators in spectral $\tau$-methods. In Proc. Third Intl Conf. on Spectral and High Order Methods (ed. A. V. Ilin \& L. Ridgway Scott). Houston Journal of Mathematics, University of Houston, pp. 21-38.

Coutsias, E. A., Hansen, F. R., Huld, T., Knorr, G. \& Lynov, J. P. 1989 Spectral methods in numerical plasma simulation. Physica Scripta 40, 270-279.

Coutsias, E. A., Hesthaven, J. S. \& Lynov, J. P. 1996 b An accurate and efficient spectral Tau method for the incompressible Navier-Stokes equations in a plane channel. In Proc. Third Intl Conf. on Spectral and High Order Methods (ed. A. V. Ilin \& L. Ridgway Scott). Houston Journal of Mathematics, University of Houston, pp. 39-54.

Coutsias, E. A. \& Lynov, J. P. 1991 Fundamental interactions of vortical structures with boundary layers in two-dimensional flows. Physica D 51, 482-497.

Don, W. S. \& Solomonoff, A 1995 Accuracy and speed in computing the Chebyshev collocation derivative. SIAM J. Sci. Comput. 16, 1253-1268.

Dovshenko, V. A. \& Krymov, V. A. 1987 Experimental study of instability of zonal shear flows in a circular geometry. Izv. Atmos. Oceanic Phys. 23, 14-20.

Drazin, P. G. \& ReID, W. H. 1991 Hydrodynamic Stability. Cambridge.

Driscoll, C. F. \& Fine, K. S. 1990 Experiments on vortex dynamics in pure electron plasmas. Phys. Fluids B 2, 1359-1366.

Gottlieb, D. \& Orszag, S. A. 1977 Numerical Analysis of Spectral Methods. SIAM.

Hide, R. \& Titman, C. W. 1967 Detached shear layers in a rotating fluid. J. Fluid Mech. 29, 39-60.

Huld, T., Nielsen, A. H., Pécseli, H. L. \& Rasmussen, J. J. 1991 Coherent structures in twodimensional plasma turbulence. Phys. Fluids B 3, 1609-1625.

Karniadakis, G. E., Israeli, M. \& Orszag, S. A. 1991 High-order splitting methods for the incompressible Navier-Stokes equations. J. Comput. Phys. 97, 414-443.

Konijnenberg, J. A. van De, Nielsen, A. H., Rasmussen, J. J. \& Stenum, B. 1999 Shear flow instability in a rotating fluid. J. Fluid Mech. 387, 177-204.

Krymov, V. A. 1988 Experimental investigation of supercritical vortex regimes in an azimuthally symmetric shear flow. Izv. Atmos. Oceanic Phys. 24, 475-482. 
Marcus, P. S. 1990 Vortex dynamics in a shearing zonal flow. J. Fluid Mech. 215, 393-430.

Maslowe, S. A. 1977 Weakly nonlinear stability of a viscous shear layer. J. Fluid Mech. 79, 689-702.

MichalKe, A. 1964 On the inviscid instability of the hyperbolic tangent velocity profile. J. Fluid Mech. 19, 543-556.

Nezlin, M. V. \& Snezhrin, E. N. 1993 Rossby Vortices, Spiral Structures, Solitons - Astrophysics and Plasma Experiments in Shallow Water Experiments. Springer.

NIELSEN, A. H. 1993 Electrostatic turbulence in strongly magnetized plasmas. PhD Thesis, Technical University of Denmark. Risø-R-659.

NiINo, H. 1982 A weakly non-linear theory of barotropic instability. J. Met. Soc. Japan 60, 10231033.

Nirno, H. \& MisaWA, N. 1984 An experimental and theoretical study of barotropic instability. J. Atmos. Sci. 41, 1992-2011.

Pécseli, H. L., Coutsias, E. A., Huld, T., Lynov, J. P., Nielsen, A. H. \& Rasmussen, J. J. 1992 Coherent vortical structures in two-dimensional plasma turbulence. Plasma Phys. Contr. Fusion 34, 2065-2070.

Pedlosky, J. 1987 Geophysical Fluid Dynamics. Springer.

Peurrung, A. J. \& FAJANS, J. 1993 Experimental dynamics of an annulus of vorticity in a pure electron plasma. Phys. Fluids A 5, 493-499.

Rabaud, M. \& Couder, Y. 1983 A shear-flow instability in a circular geometry. J. Fluid Mech. 136, 291-319.

RAND, D. 1982 Dynamics and symmetry: predictions for modulated waves in rotating fluids. Arch Rat. Mech. Anal. 79, 1-37.

Reddy, S. C., Schmid, P. J. \& Henningson, D. S. 1993 Pseudospectra of the Orr-Sommerfeld Operator. SIAM J. Appl. Math. 53, 15-47.

Solomon, T. H., Holloway, W. J. \& Swinney, H. L. 1993 Shear flow instabilities and Rossby waves in barotropic flow in a rotating annulus. Phys. Fluids A 5, 1971-1982.

Stakgold, I. 1975 Green's Functions and Boundary Value Problems. Wiley.

Tomasini, M., Dolez, N. \& LeORAT, J. 1996 Instability of a rotating shear layer in the transonic regime. J. Fluid Mech. 306, 59-82.

Wiggins, S. 1992 Chaotic Transport in Dynamical Systems. Springer. 